\title{
A QUASI-DUAL LAGRANGE MULTIPLIER SPACE FOR SERENDIPITY MORTAR FINITE ELEMENTS IN 3D*
}

\author{
Bishnu P. LAmichHane ${ }^{1}$ AND Barbara I. WOHLmuth ${ }^{1}$
}

\begin{abstract}
Domain decomposition techniques provide a flexible tool for the numerical approximation of partial differential equations. Here, we consider mortar techniques for quadratic finite elements in 3D with different Lagrange multiplier spaces. In particular, we focus on Lagrange multiplier spaces which yield optimal discretization schemes and a locally supported basis for the associated constrained mortar spaces in case of hexahedral triangulations. As a result, standard efficient iterative solvers as multigrid methods can be easily adapted to the nonconforming situation. We present the discretization errors in different norms for linear and quadratic mortar finite elements with different Lagrange multiplier spaces. Numerical results illustrate the performance of our approach.
\end{abstract}

Mathematics Subject Classification. 35N55, 65N30.

Received: May 20, 2003.

\section{INTRODUCTION}

The coupling of different discretization schemes or of nonmatching triangulations can be analyzed within the framework of mortar methods. These nonconforming domain decomposition techniques provide a more flexible approach than standard conforming approaches. The nonconforming approach is of particular interest in many situations, for example, in problems with discontinuous diffusion coefficients and local anisotropies, when different parameters dominate different parts of the simulation domain or different discretization schemes are used in different subdomains. A complex global domain can be decomposed into several small subdomains of simple structure, and these subdomains can be meshed independently. To obtain a stable and optimal discretization scheme for the global problem, the information transfer among the subdomains has to be analyzed. Mortar methods were originally introduced to couple spectral and finite element approximations, see [4,5]. An optimal a priori estimate in the $H^{1}$-norm for the mortar finite element method has been established in $[2,3,5,6]$. The analysis of three-dimensional mortar finite elements is given in [3,7,15], and a $h p$ version is studied in [17]. In the linear 3D case, the stability of the mortar projection for the standard Lagrange multiplier space is established in [7], where also a multigrid method for the saddle point problem is discussed. The main idea of the mortar technique is to replace the strong continuity condition of the solution across the interface by a weak

\footnotetext{
Keywords and phrases. Mortar finite elements, Lagrange multiplier, dual space, domain decomposition, nonmatching triangulation.

* This work was supported in part by the Deutsche Forschungsgemeinschaft, SFB 404, C12.

1 Institute of Applied Analysis and Numerical Simulation, University of Stuttgart, Germany.

e-mail: lamichhane@mathematik.uni-stuttgart.de; wohlmuth@mathematik.uni-stuttgart.de
} 
one. Here, we consider mortar methods for second order finite elements in 3D. We focus on Lagrange multiplier spaces for serendipity elements which yield locally supported basis functions of the constrained mortar space.

The paper is organized as follows: in the rest of this section, we present our model problem and briefly review the mortar method. In Section 2, we give some sufficient conditions on Lagrange multiplier spaces for quadratic finite elements and show the optimality of the approach. In Section 3, we present some examples of Lagrange multiplier spaces for quadratic finite elements on hexahedral triangulations. In contrast to earlier approaches, we use Lagrange multiplier spaces yielding a sparse inverse of the mass matrix. We also use the so-called dual Lagrange multiplier spaces which are biorthogonal to the trace of the finite element space at the interface. Unfortunately, a locally defined dual Lagrange multiplier space containing the bilinear hat functions does not exist for serendipity elements. In that case, we augment the space by face bubble functions and introduce a quasi-dual Lagrange multiplier space yielding a sparse inverse mass matrix. Finally in Section 4, we present some numerical results in 3D for different Lagrange multiplier spaces illustrating the flexibility and performance of our approach. In particular, we consider the discretization errors in the $L^{2}$-norm, the energy norm and in a weighted $L^{2}$-norm for the Lagrange multiplier.

We consider the following elliptic second order boundary value problem

$$
-\operatorname{div}(a \nabla u)+c u=f \quad \text { in } \Omega \quad \text { with } \quad u=0 \quad \text { on } \partial \Omega,
$$

where $0<a_{0} \leq a \in L^{\infty}(\Omega), f \in L^{2}(\Omega), 0 \leq c \in L^{\infty}(\Omega)$, and $\Omega \subset \mathbb{R}^{3}$ is a bounded polyhedral domain. The domain $\Omega$ is decomposed into $K$ non-overlapping polyhedral subdomains $\Omega_{k}, k=1, \cdots, K$, such that

$$
\bar{\Omega}=\bigcup_{k=1}^{K} \bar{\Omega}_{k} \quad \text { with } \quad \Omega_{i} \cap \Omega_{j}=\emptyset \quad \text { for } \quad i \neq j .
$$

Here, we consider only geometrically conforming situations where the intersection between the boundaries of any two different subdomains $\partial \Omega_{l} \cap \partial \Omega_{k}, k \neq l$, is either empty, a common edge or a face. We define $\bar{\Gamma}_{k l}:=\partial \Omega_{k} \cap \partial \Omega_{l}, 1 \leq k, l \leq K$, the intersection of the boundaries of two subdomains and select only disjoint and non-empty interfaces $\gamma_{k}, 1 \leq k \leq N$. Moreover, each $\gamma_{k}$ can be associated with a couple $1 \leq k_{1}<k_{2} \leq K$ such that $\bar{\gamma}_{k}=\partial \Omega_{k_{1}} \cap \partial \Omega_{k_{2}}$. On each subdomain, we define

$$
H_{*}^{1}\left(\Omega_{k}\right):=\left\{v \in H^{1}\left(\Omega_{k}\right), v_{\mid \partial \Omega \cap a \Omega_{k}}=0\right\}, \quad k=1, \cdots, K
$$

and consider the unconstrained product space $X:=\prod_{k=1}^{K} H_{*}^{1}\left(\Omega_{k}\right)$. The weak matching condition on the skeleton $\Gamma:=\bigcup_{k=1}^{N} \gamma_{k}$ is realized by means of the $H^{1 / 2}$-duality pairing. Introducing the Lagrange multiplier space $M:=\prod_{k=1}^{N} H^{-1 / 2}\left(\gamma_{k}\right)$ on $\Gamma$, we find for $v \in H_{0}^{1}(\Omega)$

$$
\int_{\Gamma}[v] \mu \mathrm{d} \sigma=0, \quad \mu \in M .
$$

This observation is the motivation for the discrete mortar formulation. Each subdomain $\Omega_{k}$ is associated with a shape regular family of hexahedral triangulations $\mathcal{T}_{k ; h_{k}}$, the meshsize of which is bounded by $h_{k}$. We denote the discrete space of conforming piecewise triquadratic finite elements or of serendipity elements on $\Omega_{k}$ associated with $\mathcal{T}_{k ; h_{k}}$ by $X_{h_{k}} \subset H_{*}^{1}\left(\Omega_{k}\right)$. Each interface $\gamma_{k}$ inherits a two-dimensional triangulation $\mathcal{S}_{k ; h_{k}}$ either from $\mathcal{T}_{k_{1} ; h_{k_{1}}}$ or $\mathcal{T}_{k_{2} ; h_{k_{2}}}$. The subdomain from which the interface inherits its triangulation is called slave or nonmortar side, the opposite one master or mortar side. In the following, we denote the index of the slave side of $\gamma_{k}$ by $s(k)$ and the one of the master side by $m(k)$. Hence, the elements of $\mathcal{S}_{k ; h_{k}}$ are boundary faces of $\mathcal{T}_{s(k) ; h_{s(k)}}$ with a meshsize bounded by $h_{s(k)}$. Furthermore, we assume that the mesh on $\Gamma$ is globally quasi-uniform, and each element in $\mathcal{S}_{k ; h_{k}}, k=1, \cdots, N$, can be affinely mapped to the reference element $\hat{T}:=(0,1) \times(0,1)$. The 
discrete Lagrange multiplier space $M_{h}$ on $\Gamma$ is defined as $M_{h}:=\prod_{k=1}^{N} M_{h}\left(\gamma_{k}\right)$, where $M_{h}\left(\gamma_{k}\right)$ is the discrete Lagrange multiplier space on $\gamma_{k}$. Then, the discrete weak matching condition for $v_{h} \in X_{h}$ can be written as

$$
\int_{\gamma_{k}}\left[v_{h}\right] \mu_{i} \mathrm{~d} \sigma=0, \quad 1 \leq i \leq n_{k}, 1 \leq k \leq N
$$

where $n_{k}:=\operatorname{dim} M_{h}\left(\gamma_{k}\right)$ and $\left\{\mu_{i}\right\}_{1 \leq i \leq n_{k}}$ forms a basis of $M_{h}\left(\gamma_{k}\right)$. Here, $\left[v_{h}\right]$ is the jump of the function $v_{h}$ on $\gamma_{k}$ from the master side to the slave side. As usual, $\|\cdot\|_{s, \Omega_{k}}$ and $(\cdot, \cdot)_{s, \Omega_{k}}$ denote the norm and the corresponding inner product on $H^{s}\left(\Omega_{k}\right)$, respectively, and $|\cdot|_{s, \Omega_{k}}$ stands for the seminorm. The norm on $H_{00}^{1 / 2}\left(\gamma_{k}\right)$ and its dual space $H^{-1 / 2}\left(\gamma_{k}\right)$ will be denoted by $\|\cdot\|_{H_{00}^{1 / 2}\left(\gamma_{k}\right)}$ and $\|\cdot\|_{-1 / 2, \gamma_{k}}$, respectively. We define the broken norm $\|\cdot\|_{s}$ on $X$ and the broken dual norm $\|\cdot\|_{M}$ on $M$ by

$$
\|u\|_{s}^{2}:=\sum_{k=1}^{K}\|u\|_{s, \Omega_{k}}^{2}, \quad \text { and } \quad\|\mu\|_{M}^{2}:=\sum_{k=1}^{N}\|\mu\|_{-1 / 2, \gamma_{k}}^{2}, \quad \text { respectively. }
$$

There are two main approaches to obtain the mortar solution $u_{h} \in X_{h}$ of a discrete variational problem. The first one is based on the positive definite variational problem on the constrained finite element space which is given by means of the global Lagrange multiplier space $M_{h}$

$$
V_{h}:=\left\{v_{h} \in X_{h} \mid b\left(v_{h}, \mu_{h}\right)=0, \mu_{h} \in M_{h}\right\}
$$

where $\quad b\left(v_{h}, \mu_{h}\right):=\sum_{k=1}^{N} \int_{\gamma_{k}}\left[v_{h}\right] \mu_{h} d \sigma$, and $X_{h}:=\prod_{k=1}^{K} X_{h_{k}}$. We remark that the elements of the space $V_{h}$ satisfy a weak continuity condition on the skeleton $\Gamma$ in terms of the discrete Lagrange multiplier space $M_{h}$, and the nodal basis functions of $X_{h}$ have to be modified appropriately to obtain the basis functions of $V_{h}$. However, $V_{h}$ is, in general, not a subspace of $H_{0}^{1}(\Omega)$. The positive definite formulation of the mortar method can be given in terms of the constrained space $V_{h}$ : find $u_{h} \in V_{h}$ such that

$$
a\left(u_{h}, v_{h}\right)=\left(f, v_{h}\right)_{0}, \quad v_{h} \in V_{h}
$$

where, the bilinear form $a(\cdot, \cdot)$ is defined as $a(v, w):=\sum_{k=1}^{K} \int_{\Omega_{k}} a \nabla v \cdot \nabla w+c v w \mathrm{~d} x$. The second approach is based on enforcing the weak continuity condition on the skeleton $\Gamma$ as an additional variational equation which leads to a saddle point problem on the unconstrained product space $X_{h}$, see [2]: find $\left(u_{h}, \lambda_{h}\right) \in X_{h} \times M_{h}$ such that

$$
\begin{aligned}
a\left(u_{h}, v_{h}\right)+b\left(v_{h}, \lambda_{h}\right) & =\left(f, v_{h}\right)_{0}, & & v_{h} \in X_{h}, \\
b\left(u_{h}, \mu_{h}\right) & =0, & & \mu_{h} \in M_{h} .
\end{aligned}
$$

It is clear that the choice of the discrete Lagrange multiplier space $M_{h}$ plays an essential role for the stability of the saddle point problem and the optimality of the discretization scheme. In the next section, we state sufficient conditions on the Lagrange multiplier space for quadratic finite elements to get optimal a priori estimates. Here, the nodal Lagrange multiplier basis functions are defined locally and are associated with the interior nodes of the mesh on $\gamma_{k}, k=1, \cdots, N$. We point out that we do not assume the meshes from the slave and master side are matching on $\partial \gamma_{k}$, see Figure 1. Now, we group the degrees of freedom of $X_{h}$ associated with the skeleton $\Gamma$ into two groups $u_{\left.h\right|_{\Gamma}}:=\left(u_{m}, u_{s}\right)$, where $u_{m}$ contains all nodal values of $u_{h}$ on the master sides and all nodal values on the boundary of the interface $\gamma_{k}$ on the slave sides, and $u_{s}$ consists of all nodal values of $u_{h}$ at the interior nodes of $\gamma_{k}$ on the slave sides, $1 \leq k \leq N$, see Figure 1 . The associated sets of nodes are called $\mathcal{N}_{m}$ and $\mathcal{N}_{s}$, respectively. Furthermore, we denote by $\mathcal{N}_{h}$ the set of all nodes in $X_{h}$ and we set $\mathcal{N}_{i}:=\mathcal{N}_{h} \backslash\left(\mathcal{N}_{m} \cup \mathcal{N}_{s}\right)$. The corresponding nodal values of $u_{h}$ in $\mathcal{N}_{i}$ will be denoted by a block vector $u_{i}$. Then, (1.2) can be written in its algebraic form as

$$
M_{s} u_{s}+M_{m} u_{m}=0
$$



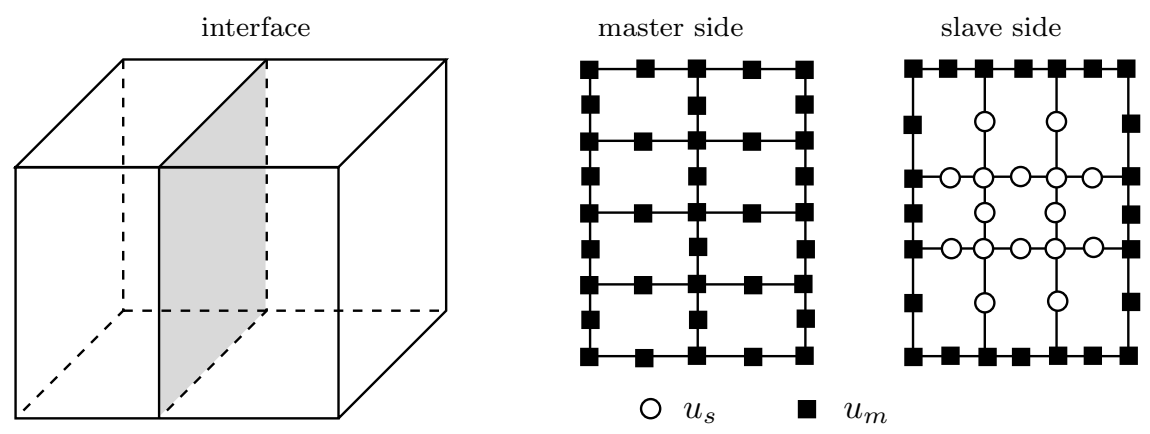

Figure 1. Decomposition into $u_{m}$ and $u_{s}$ for serendipity elements.

The entries of the mass matrices are given by $m_{i j}:=\int_{\gamma_{k}}\left[\phi_{j}\right] \mu_{i} \mathrm{~d} \sigma$, where $\phi_{j}$ are the finite element basis functions corresponding to the different groups of nodes, and $\mu_{i}$ denote the basis functions of $M_{h}$. Since the basis functions have a local support, the mass matrices are sparse. Formally, we can obtain the values on the slave side as $u_{s}=-M_{s}^{-1} M_{m} u_{m}$. Although $M_{s}$ is a sparse matrix, the inversion of $M_{s}$ is, in general, expensive, and $M_{s}^{-1}$ is dense. This observation motivates our interest in Lagrange multiplier spaces which yield a sparse inverse of the mass matrix $M_{s}$. A natural choice is a dual Lagrange multiplier space, see, e.g., [21], having a diagonal mass matrix $M_{s}$. Then, the basis functions $\left\{\mu_{i}\right\}_{1 \leq i \leq n_{k}}$ of $M_{h}\left(\gamma_{k}\right)$ and $\left\{\varphi_{i}\right\}_{1 \leq i \leq n_{k}}$ of the trace space $W_{0, h}\left(\gamma_{k}\right)$ having the zero boundary condition on $\partial \gamma_{k}$ satisfy the biorthogonality relation

$$
\int_{\gamma_{k}} \mu_{i} \varphi_{j} \mathrm{~d} \sigma=\delta_{i j} \int_{\gamma_{k}} \varphi_{j} \mathrm{~d} \sigma, \quad 1 \leq i, j \leq n_{k}
$$

We define the product space $W_{0, h}$ and the broken $H_{00}^{1 / 2}$-norm on it as

$$
W_{0, h}:=\prod_{k=1}^{N} W_{0, h}\left(\gamma_{k}\right), \quad \text { and } \quad\|v\|_{W}^{2}:=\sum_{k=1}^{N}\|v\|_{H_{00}^{1 / 2}\left(\gamma_{k}\right)}^{2}, \quad \text { respectively. }
$$

\section{A PRIORI ESTimates}

In this section, we give some assumptions on quadratic Lagrange multiplier spaces which guarantee optimal a priori estimates. Following a similar approach as in [15], we impose the following assumptions on the discrete Lagrange multiplier spaces for quadratic finite elements

[P0] $\operatorname{dim} M_{h}\left(\gamma_{k}\right)=\operatorname{dim} W_{0, h}\left(\gamma_{k}\right), \quad 1 \leq k \leq N$.

[P1] There is a constant $C$ independent of the triangulation such that

$$
\inf _{\mu \in M_{h}\left(\gamma_{k}\right)}\|v-\mu\|_{0, \gamma_{k}} \leq C h_{s(k)}^{2}|v|_{2, \gamma_{k}}, \quad v \in H^{2}\left(\gamma_{k}\right), \quad 1 \leq k \leq N
$$

[P2 ] There is a constant $C$ independent of the triangulation such that

$$
\|\theta\|_{0, \gamma_{k}} \leq C \sup _{\mu \in M_{h}\left(\gamma_{k}\right) \backslash\{0\}} \frac{(\theta, \mu)_{0, \gamma_{k}}}{\|\mu\|_{0, \gamma_{k}}}, \quad \theta \in W_{0, h}\left(\gamma_{k}\right), \quad 1 \leq k \leq N .
$$


It follows from assumption $[\mathrm{P} 1]$ that $\mathcal{P}_{1}\left(\gamma_{k}\right) \subset M_{h}\left(\gamma_{k}\right)$ for all $k=1, \cdots, N$, where $\mathcal{P}_{1}\left(\gamma_{k}\right)$ is the space of linear functions on $\gamma_{k}$. For each $\gamma_{k}$, the mortar projection $\Pi_{k}: L^{2}\left(\gamma_{k}\right) \rightarrow W_{0, h}\left(\gamma_{k}\right)$ is defined as

$$
\int_{\gamma_{k}} \Pi_{k} v \mu \mathrm{d} \sigma:=\int_{\gamma_{k}} v \mu \mathrm{d} \sigma, \quad \mu \in M_{h}\left(\gamma_{k}\right) .
$$

The stability of the mortar projection is essential for the optimality of the best approximation error.

Lemma 2.1. Under the assumptions [P0] and [P2], the mortar projection (2.1) is stable in the $L^{2}-$ norm. Furthermore, if $w \in H_{0}^{1}\left(\gamma_{k}\right)$

$$
\left\|\Pi_{k} w\right\|_{1, \gamma_{k}} \leq C\|w\|_{1, \gamma_{k}}
$$

Proof. By assumption [P2], we find that if $v \in W_{0, h}\left(\gamma_{k}\right)$ satisfies $(v, \mu)_{0, \gamma_{k}}=0$ for all $\mu \in M_{h}\left(\gamma_{k}\right)$, then $v=0$. Hence, the mortar projection is well-defined by the assumptions [P0] and [P2]. The $L^{2}$-stability of $\Pi_{k}$ is standard, see, e.g., [15]. Now for $w \in H_{0}^{1}\left(\gamma_{k}\right)$ using the $L^{2}$-stability and an inverse estimate, we find

$$
\begin{aligned}
\left\|\Pi_{k} w\right\|_{1, \gamma_{k}} \leq\left\|\Pi_{k} w-P w\right\|_{1, \gamma_{k}}+\|P w\|_{1, \gamma_{k}} & \leq C\left(\frac{1}{h_{s(k)}}\left\|\Pi_{k}(w-P w)\right\|_{0, \gamma_{k}}+\|w\|_{1, \gamma_{k}}\right) \\
& \leq C\left(\frac{1}{h_{s(k)}}\|w-P w\|_{0, \gamma_{k}}+\|w\|_{1, \gamma_{k}}\right) \leq C\|w\|_{1, \gamma_{k}},
\end{aligned}
$$

where $P$ denotes the $L^{2}$-projection onto $W_{0, h}\left(\gamma_{k}\right)$.

Using Lemma 2.1 and an interpolation argument, we obtain for $w \in H_{00}^{1 / 2}\left(\gamma_{k}\right)$,

$$
\left\|\Pi_{k} w\right\|_{H_{00}^{1 / 2}\left(\gamma_{k}\right)} \leq C\|w\|_{H_{00}^{1 / 2}\left(\gamma_{k}\right)} .
$$

In a next step, we provide the best approximation property of the space $V_{h}$. We use the ideas and techniques introduced in $[3,5]$.

Lemma 2.2. Assume that the assumptions [P0]-[P2] hold. If $u \in H_{0}^{1}(\Omega)$ and $u_{\mid \Omega_{k}} \in H^{3}\left(\Omega_{k}\right)$ for all $k=$ $1, \cdots, K$, then there exists a constant $C$ independent of the meshsizes such that

$$
\inf _{u_{h} \in V_{h}}\left\|u-u_{h}\right\|_{1}^{2} \leq C\left(1+h_{m r}\right) \sum_{k=1}^{K} h_{k}^{4}\|u\|_{3, \Omega_{k}}^{2}
$$

where

$$
h_{m r}:=\max \left\{\frac{h_{m(k)}}{h_{s(k)}}, \quad 1 \leq k \leq N\right\} .
$$

Proof. Since $W_{0, h}\left(\gamma_{k}\right) \subset H_{00}^{1 / 2}\left(\gamma_{k}\right)$, each $v \in W_{0, h}\left(\gamma_{k}\right)$ can trivially be extended to a function $\tilde{v} \in H^{1 / 2}\left(\partial \Omega_{s(k)}\right)$. Let $\mathcal{H}_{h} \tilde{v} \in H^{1}\left(\Omega_{s(k)}\right)$ be the discrete harmonic extension of $\tilde{v}$ on $\Omega_{s(k)}$. Then, it is well known that $\left\|\mathcal{H}_{h} \tilde{v}\right\|_{1, \Omega_{s(k)}} \leq$ $C\|\tilde{v}\|_{H^{1 / 2}\left(\partial \Omega_{s(k)}\right)} \leq C\|v\|_{H_{00}^{1 / 2}\left(\gamma_{k}\right)}$. By means of this discrete harmonic extension, we define a discrete extension operator $E_{k}: W_{0, h}\left(\gamma_{k}\right) \rightarrow X_{h}$ for each $\gamma_{k}$ as $E_{k} v:=\mathcal{H}_{h} \tilde{v}$ on $\Omega_{s(k)}$, and $E_{k} v:=0$ elsewhere. Then

$$
\left\|E_{k} v\right\|_{1} \leq C\|v\|_{H_{00}^{1 / 2}\left(\gamma_{k}\right)}, \quad v \in W_{0, h}\left(\gamma_{k}\right) .
$$


Let $I_{h} u \in X_{h}$ be the Lagrange interpolant of $u$ in $X_{h}$. It is easy to see that $v:=I_{h} u+\sum_{k=1}^{N} E_{k} \Pi_{k}\left[I_{h} u\right]$ is an element of $V_{h}$. Then, we find

$$
\|u-v\|_{1} \leq\left\|u-I_{h} u\right\|_{1}+\left\|\sum_{k=1}^{N} E_{k} \Pi_{k}\left[I_{h} u\right]\right\|_{1} .
$$

By using (2.2) and a coloring argument, we have

$$
\left\|\sum_{k=1}^{N} E_{k} \Pi_{k}\left[I_{h} u\right]\right\|_{1}^{2}=\sum_{l=1}^{K}\left\|\sum_{k=1}^{N} E_{k} \Pi_{k}\left[I_{h} u\right]\right\|_{1, \Omega_{l}}^{2} \leq C \sum_{k=1}^{N}\left\|\Pi_{k}\left[I_{h} u\right]\right\|_{H_{00}^{1 / 2}\left(\gamma_{k}\right)}^{2} .
$$

We note that the constant $C$ does not depend on the number of subdomains. Applying the $L^{2}$-stability of $\Pi_{k}$ and an inverse estimate, we get

$$
\begin{aligned}
\left\|\Pi_{k}\left[I_{h} u\right]\right\|_{H_{00}^{1 / 2}\left(\gamma_{k}\right)}^{2} \leq \frac{C}{h_{s(k)}}\left\|\left[I_{h} u\right]\right\|_{0, \gamma_{k}}^{2} & \leq \frac{C}{h_{s(k)}}\left(\left\|\left(u-I_{h} u\right)_{\left.\right|_{\Omega_{m(k)}}}\right\|_{0, \gamma_{k}}^{2}+\left\|\left(u-I_{h} u\right)_{\mid \Omega_{s(k)}}\right\|_{0, \gamma_{k}}^{2}\right) \\
& \leq \frac{C}{h_{s(k)}}\left(h_{m(k)}^{5}\|u\|_{3, \Omega_{m(k)}}^{2}+h_{s(k)}^{5}\|u\|_{3, \Omega_{s(k)}}^{2}\right) .
\end{aligned}
$$

Summing over all $k=1, \cdots, N$, we obtain

$$
\left\|\sum_{k=1}^{N} E_{k} \Pi_{k}\left[I_{h} u\right]\right\|_{1}^{2} \leq C\left(h_{m r} \sum_{k=1}^{N} h_{m(k)}^{4}\|u\|_{3, \Omega_{m(k)}}^{2}+\sum_{k=1}^{N} h_{s(k)}^{4}\|u\|_{3, \Omega_{s(k)}}^{2}\right) .
$$

Finally, the lemma follows by using the interpolation property of $I_{h} u$.

We remark that in contrast to a convergence theory of mortar finite elements in $2 \mathrm{D}$, the constant in the right hand side depends on the ratio of the meshsizes of master and slave sides. This results from the fact that we cannot exploit the $H_{00}^{1 / 2}$ - stability of $\Pi_{k}$. We observe that due to the possible non-matching meshes on $\partial \gamma_{k}$, we cannot guarantee that $\left[I_{h} u\right]_{\gamma_{\gamma_{k}}}$ is in $H_{00}^{1 / 2}\left(\gamma_{k}\right)$. However, if the meshes on the wirebasket are matching and $u$ is continuous, we find $\left[I_{h} u\right]_{\left.\right|_{\gamma_{k}}} \in H_{00}^{1 / 2}\left(\gamma_{k}\right)$ and thus the $H_{00}^{1 / 2}$-stability of $\Pi_{k}$ can be directly applied. In that case, the ratio does not enter in the upper bound, see [15]. Working with mesh dependent norms and a trivial extension shows that the global ratio $h_{m r}$ can be replaced by a local one.

Theorem 2.3. Let $u$ and $u_{h}$ be the solutions of Problem (1.1) and (1.3), respectively. Assume that $u \in H_{0}^{1}(\Omega)$, $u_{\left.\right|_{\Omega_{k}}} \in H^{3}\left(\Omega_{k}\right)$ for $k=1, \cdots, K$, and $\left[a \frac{\partial u}{\partial n}\right]=0$ on $\Gamma$. Under the assumptions [P0]-[P2], there exists a constant $C$ independent of the meshsizes such that

$$
\left\|u-u_{h}\right\|_{1}^{2} \leq C\left(1+h_{m r}\right) \sum_{k=1}^{K} h_{k}^{4}\|u\|_{3, \Omega_{k}}^{2} .
$$

Proof. The bilinear form $a(\cdot, \cdot)$ is continuous on $X$, and it is coercive on

$$
B:=\left\{v \mid v \in H_{*}^{1}\left(\Omega_{k}\right), 1 \leq k \leq K, \text { and } \int_{\gamma_{k}}[v] \mathrm{d} \sigma=0,1 \leq k \leq N\right\}
$$

see $[5,14]$. Hence, assumption [P1] assures that $V_{h} \subset B$. Thus, Strang's Lemma [9] can be applied, and we get

$$
\left\|u-u_{h}\right\|_{1} \leq C\left(\inf _{v_{h} \in V_{h}}\left\|u-v_{h}\right\|_{1}+\sup _{v_{h} \in V_{h} \backslash\{0\}} \frac{\left|a\left(u-u_{h}, v_{h}\right)\right|}{\left\|v_{h}\right\|_{1}}\right) .
$$


The first term in the right side of (2.3) denotes the best approximation error and the second one stands for the consistency error. Lemma 2.2 guarantees the required order for the best approximation error. Thus it is sufficient to consider the consistency error in more detail. Now, $a\left(u-u_{h}, v_{h}\right)$ can be written as

$$
a\left(u-u_{h}, v_{h}\right)=\int_{\Gamma} a \frac{\partial u}{\partial n}\left[v_{h}\right] \mathrm{d} \sigma=\sum_{k=1}^{N}\left(a \frac{\partial u}{\partial n_{k}},\left[v_{h}\right]\right)_{0, \gamma_{k}}, \quad v_{h} \in V_{h}
$$

Here, $\frac{\partial u}{\partial n}$ is the outward normal derivative of $u$ on $\Gamma$ from the master side, and $\frac{\partial u}{\partial n}=\frac{\partial u}{\partial n_{k}}$ on $\gamma_{k}$. Using the definition of $V_{h}$, we find for $\mu \in M_{h}\left(\gamma_{k}\right)$

$$
\left(a \frac{\partial u}{\partial n_{k}},\left[v_{h}\right]\right)_{0, \gamma_{k}}=\left(a \frac{\partial u}{\partial n_{k}}-\mu,\left[v_{h}\right]\right)_{0, \gamma_{k}} \leq \inf _{\mu \in M_{h}\left(\gamma_{k}\right)}\left\|a \frac{\partial u}{\partial n_{k}}-\mu\right\|_{\left(H^{1 / 2}\left(\gamma_{k}\right)\right)^{\prime}}\left\|\left[v_{h}\right]\right\|_{1 / 2, \gamma_{k}} .
$$

Due to assumption [P1], we find that the best approximation error of $M_{h}\left(\gamma_{k}\right)$ in the $H^{1 / 2}$-dual norm is bounded by $C h_{s(k)}^{2}\left\|\frac{\partial u}{\partial n_{k}}\right\|_{3 / 2, \gamma_{k}}$. We note that the $H^{1 / 2}$-dual norm is stronger than the $H^{-1 / 2}$-norm. Now the trace theorem yields the upper bound for the consistency error

$$
\left(a \frac{\partial u}{\partial n_{k}},\left[v_{h}\right]\right)_{0, \gamma_{k}} \leq C h_{s(k)}^{2}\|u\|_{3, \Omega_{s(k)}}\left(\left\|v_{h}\right\|_{1, \Omega_{m(k)}}+\left\|v_{h}\right\|_{1, \Omega_{s(k)}}\right) .
$$

See [15, Theorem 3.1] for the linear case. Now, using the Cauchy-Schwarz inequality and summing over all $k=1, \cdots, N$, we find for $v_{h} \in V_{h}$

$$
\left|a\left(u-u_{h}, v_{h}\right)\right| \leq C\left\|v_{h}\right\|_{1}\left(\sum_{k=1}^{K} h_{k}^{4}\|u\|_{3, \Omega_{k}}^{2}\right)^{1 / 2} .
$$

To obtain an a priori estimate for the Lagrange multipliers, we follow exactly the same lines as in [2].

Lemma 2.4. Assume that the Lagrange multiplier space $M_{h}$ satisfies the assumptions [P0]-[P2]. Then for $\mu \in M_{h}$, there exists a $v_{\mu} \in X_{h}$ such that

$$
\left\|v_{\mu}\right\|_{1} \leq C\|\mu\|_{M}, \quad\|\mu\|_{M}^{2} \leq C b\left(v_{\mu}, \mu\right) \quad \text { and } \quad\left\|\left[v_{\mu}\right]\right\|_{W} \leq C\|\mu\|_{M} .
$$

Proof. By means of the stability of the mortar projection, we get for $\mu \in M_{h}\left(\gamma_{k}\right)$

$$
\begin{aligned}
\|\mu\|_{-1 / 2, \gamma_{k}} & =\sup _{\varphi \in H_{00}^{1 / 2}\left(\gamma_{k}\right) \backslash\{0\}} \frac{(\mu, \varphi)_{0, \gamma_{k}}}{\|\varphi\|_{H_{00}^{1 / 2}\left(\gamma_{k}\right)}} \leq C \sup _{\varphi \in H_{00}^{1 / 2}\left(\gamma_{k}\right) \backslash\{0\}} \frac{\left(\mu, \Pi_{k} \varphi\right)_{0, \gamma_{k}}}{\left\|\Pi_{k} \varphi\right\|_{H_{00}^{1 / 2}\left(\gamma_{k}\right)}} \\
& =C \sup _{\varphi \in W_{0, h}\left(\gamma_{k}\right) \backslash\{0\}} \frac{(\mu, \varphi)_{0, \gamma_{k}}}{\|\varphi\|_{H_{00}^{1 / 2}\left(\gamma_{k}\right)}} \leq C\left(\mu, \tilde{\varphi}_{k}\right)_{0, \gamma_{k}}
\end{aligned}
$$

for some $\tilde{\varphi}_{k} \in W_{0, h}\left(\gamma_{k}\right)$ with $\left\|\tilde{\varphi}_{k}\right\|_{H_{00}^{1 / 2}\left(\gamma_{k}\right)}=1$. Now, we extend $\tilde{\varphi}_{k} \in W_{0, h}\left(\gamma_{k}\right)$ to $X_{h}$ by using the extension operator $E_{k}$ as defined in Lemma 2.2 to get $E_{k} \tilde{\varphi}_{k}=: v_{k} \in X_{h}$. Then, we have

$$
\left\|v_{k}\right\|_{1} \leq C\left\|\tilde{\varphi}_{k}\right\|_{H_{00}^{1 / 2}\left(\gamma_{k}\right)} \text { and } \quad 0 \leq\left(\mu, \tilde{\varphi}_{k}\right)_{0, \gamma_{k}}=b\left(v_{k}, \mu\right) .
$$

Setting $v_{\mu}:=\sum_{k=1}^{N} b\left(v_{k}, \mu\right) v_{k}$ and using the fact that $\left\|v_{k}\right\|_{1} \leq C$, we get

$$
\left\|v_{\mu}\right\|_{1}^{2}=\sum_{l=1}^{K}\left\|\sum_{k=1}^{N} b\left(v_{k}, \mu\right) v_{k}\right\|_{1, \Omega_{l}}^{2} \leq C \sum_{k=1}^{N} b\left(v_{k}, \mu\right)^{2} \leq C \sum_{k=1}^{N}\|\mu\|_{-1 / 2, \gamma_{k}}^{2}=C\|\mu\|_{M}^{2} .
$$


To obtain the upper bound for $\|\mu\|_{M}$, we sum the equation (2.4) over all interfaces $\gamma_{k}, k=1, \cdots, N$ and find

$$
\|\mu\|_{M}^{2} \leq C \sum_{k=1}^{N} b\left(v_{k}, \mu\right)^{2}=C b\left(v_{\mu}, \mu\right)
$$

Finally, the third assertion follows from

$$
\left\|\left[v_{\mu}\right]\right\|_{W}^{2}=\sum_{k=1}^{N} b\left(v_{k}, \mu\right)^{2}\left\|\left[v_{k}\right]\right\|_{H_{00}^{1 / 2}\left(\gamma_{k}\right)}^{2}=\sum_{k=1}^{N} b\left(v_{k}, \mu\right)^{2} \leq \sum_{k=1}^{N}\|\mu\|_{-1 / 2, \gamma_{k}}^{2}\left\|\left[v_{k}\right]\right\|_{H_{00}^{1 / 2}\left(\gamma_{k}\right)}^{2}=\|\mu\|_{M}^{2} .
$$

We note that the bilinear form $b(\cdot, \cdot)$ on $X_{h} \times M_{h}$ is not continuous with respect to the $\|\cdot\|_{1}$ and $\|\cdot\|_{M}$ norm. However the uniform inf-sup condition for $\mu_{h} \in M_{h}$ can be established on a subspace of $X_{h}$. Restricted to this subspace the bilinear form $b(\cdot, \cdot)$ is continuous and thus the standard saddle point theory can be applied, see, e.g., [10]. Combining the previous results, an a priori bound for the Lagrange multiplier can be obtained.

Corollary 2.5. Under the assumptions of Theorem 2.3, we have

$$
\left\|\lambda-\lambda_{h}\right\|_{M}^{2} \leq C \sum_{k=1}^{K} h_{k}^{4}\|u\|_{3, \Omega_{k}}^{2}
$$

\section{QuAdratic Lagrange MUltiplier SPACES IN 3D}

In this section, we consider different possibilities for Lagrange multiplier spaces in 3D for quadratic finite elements with $\operatorname{supp} \varphi_{\mathrm{i}}=\operatorname{supp} \mu_{\mathrm{i}}$. In particular, we focus on the standard finite elements and serendipity elements and restrict ourselves to hexahedral triangulations. These two finite element spaces have different degrees of freedom on the interface and therefore, the Lagrange multiplier spaces have to be considered separately.

\subsection{A dual Lagrange multiplier space for triquadratic finite elements}

In the case of a hexahedral triangulation, a dual Lagrange multiplier space in 3D for trilinear and triquadratic finite elements can be formed by taking the tensor product of the dual Lagrange multiplier space in 2D. Let $\hat{\varphi}_{0}, \hat{\varphi}_{1}$ and $\hat{\varphi}_{2}$ be the nodal quadratic finite element basis functions on the reference element $(0,1)$ in one dimension, where $\hat{\varphi}_{0}$ and $\hat{\varphi}_{1}$ are the basis functions corresponding to the left and the right vertices of the reference element, and $\hat{\varphi}_{2}$ is the basis function corresponding to the midpoint of the reference element. Then, the quadratic dual Lagrange multiplier basis functions on the reference element are defined by

$$
\hat{\lambda}_{0}(t):=\hat{\varphi}_{0}(t)-\frac{3}{4} \hat{\varphi}_{2}(t)+\frac{1}{2}, \quad \hat{\lambda}_{1}(t):=\hat{\varphi}_{1}(t)-\frac{3}{4} \hat{\varphi}_{2}(t)+\frac{1}{2} \quad \text { and } \quad \hat{\lambda}_{2}(t):=\frac{5}{2} \hat{\varphi}_{2}(t)-1 .
$$

The Lagrange multiplier basis functions for the element touching a crosspoint have to be modified. In particular, if $t=0$ is a crosspoint, we have

$$
\hat{\lambda}_{2}(t):=-2 t+2, \quad \hat{\lambda}_{1}(t):=2 t-1,
$$

and if $t=1$ is a crosspoint, we set

$$
\hat{\lambda}_{2}(t):=2 t, \quad \hat{\lambda}_{0}(t):=1-2 t .
$$

Furthermore, for a linear hat function $\phi_{p}^{l}$ at an interior vertex $p$, we find

$$
\phi_{p}^{l}(t)=\mu_{p}(t)+\frac{1}{2}\left(\mu_{e 1}(t)+\mu_{e 2}(t)\right)
$$



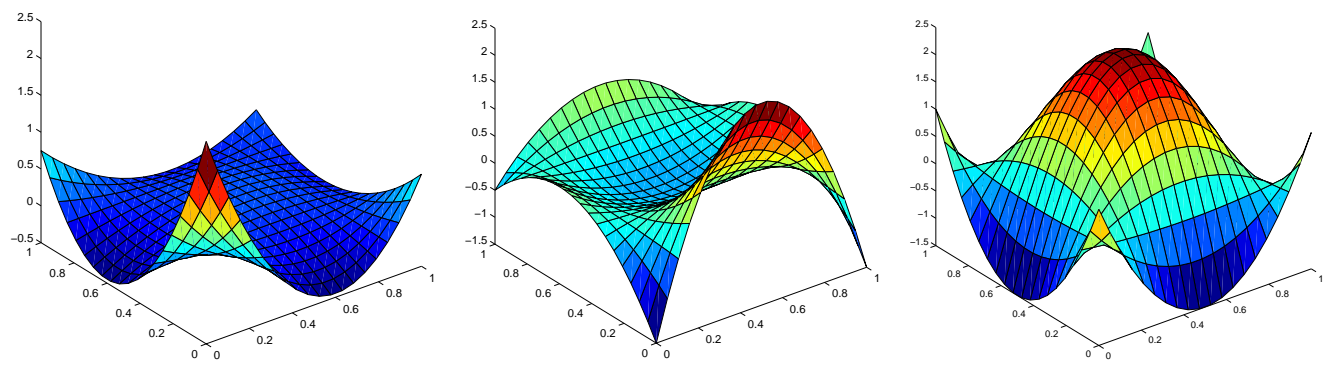

Figure 2. The Lagrange multipliers corresponding to a vertex (left), to an edge (middle) and to the center of gravity (right).

where $\mu_{p}$ is the Lagrange multiplier basis function corresponding to the vertex $p$ and $\mu_{e 1}$ and $\mu_{e 2}$ are the basis functions associated with the midpoints of the two adjacent edges. If $p$ is a crosspoint, we have $\phi_{p}^{l}(t)=\frac{1}{2} \mu_{e}(t)$, where $\mu_{e}$ is the Lagrange multiplier basis function corresponding to the midpoint of the edge containing the crosspoint. Then, the Lagrange multiplier basis functions on the reference face $\hat{F}=(0,1) \times(0,1)$ having a tensor product structure are defined as

$$
\hat{\lambda}_{i j}(x, y):=\hat{\lambda}_{i}(x) \hat{\lambda}_{j}(y)
$$

Here, $\hat{\lambda}_{00}(x, y), \hat{\lambda}_{10}(x, y), \hat{\lambda}_{11}(x, y)$ and $\hat{\lambda}_{01}(x, y)$ are the Lagrange multipliers corresponding to the four vertices $(0,0),(1,0),(1,1)$ and $(0,1)$, and $\hat{\lambda}_{20}(x, y), \hat{\lambda}_{12}(x, y), \hat{\lambda}_{21}(x, y)$ and $\hat{\lambda}_{02}(x, y)$ are the ones corresponding to the midpoints $(0.5,0),(1,0.5),(0.5,1)$ and $(0,0.5)$ of the four edges, respectively, and finally $\hat{\lambda}_{22}(x, y)$ is the one corresponding to the center of gravity $(0.5,0.5)$ of the reference face. The Lagrange multiplier basis functions are associated with the vertices, midpoints of the edges and the center of gravity of faces in $\mathcal{S}_{k ; h_{k}}, 1 \leq k \leq N$. The global basis functions $\mu_{i}$ are obtained by using an affine mapping and gluing the local ones together. All nodes on the boundary $\partial \gamma_{k}$ of $\gamma_{k}$ are crosspoints and do not carry a degree of freedom for the Lagrange multiplier space. We note that we have to use the modification at the crosspoints to compute the tensor product for the Lagrange multipliers corresponding to the faces touching $\partial \gamma_{k}$. Observing (3.1), we find that the bilinear hat function at each vertex is contained in the Lagrange multiplier space $M_{h}\left(\gamma_{k}\right)$. We point out that this is also valid on $\partial \gamma_{k}$, although there are no degrees of freedom. Hence, assumption [P1] is satisfied. Assumption [P0] is trivially satisfied by construction. Now, we verify assumption [P2]. Let $\varphi:=\sum_{k=1}^{n_{k}} a_{k} \varphi_{k}$ be in $W_{0, h_{k}}\left(\gamma_{k}\right)$ and set $\mu:=\sum_{k=1}^{n_{k}} a_{k} \mu_{k}$. In the following, we assume that $\hat{\varphi}_{i}$ and $\hat{\mu}_{i}$ are obtained from $\varphi_{i}$ and $\mu_{i}$ by an affine mapping from the face $F$ to the reference face $\hat{F}$. Now, by using the biorthogonality relation (1.6) and the quasi-uniformity assumption, we get

$$
(\varphi, \mu)_{0, \gamma_{k}}=\sum_{i, j=1}^{n_{k}} a_{i} a_{j}\left(\varphi_{i}, \mu_{j}\right)_{0, \gamma_{k}}=\sum_{i=1}^{n_{k}} a_{i}^{2} \int_{\gamma_{k}} \varphi_{i} \mathrm{~d} \sigma \geq C \sum_{i=1}^{n_{k}} a_{i}^{2} h_{s(k)}^{2} \geq C\|\varphi\|_{0, \gamma_{k}}^{2}
$$

Taking into account the fact that $\|\varphi\|_{0, \gamma_{k}}^{2} \equiv\|\mu\|_{0, \gamma_{k}}^{2} \equiv \sum_{i=1}^{n_{k}} a_{i}^{2} h_{s(k)}^{2}$, we find that assumption [P2] is satisfied. Figure 2 shows the three different types of Lagrange multipliers on the reference face.

\subsection{A non-existence result for serendipity elements}

Here, we provide a non-existence result for a dual Lagrange multiplier space for serendipity elements. A similar result for simplicial triangulations and quadratic finite elements is given in [16]. We denote by $W_{h}^{1}\left(\gamma_{k}\right)$ the finite element space of piecewise bilinear hat functions on $\gamma_{k}$. In case of standard triquadratic finite elements, the dual Lagrange multiplier space with tensor product structure contains $W_{h}^{1}\left(\gamma_{k}\right)$. Unfortunately, there exists 


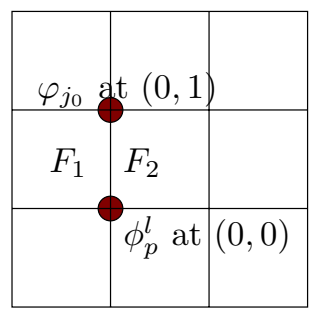

FiguRE 3. 2D interface of 3D hexahedral triangulation.

no dual Lagrange multiplier space yielding optimal a priori estimates with $\operatorname{supp} \varphi_{\mathrm{i}}=\operatorname{supp} \mu_{\mathrm{i}}$, where $\varphi_{i}$ are the serendipity nodal finite element basis functions on the interface $\gamma_{k}$.

Lemma 3.1. Under the assumption that $\operatorname{supp} \varphi_{\mathrm{i}}=\operatorname{supp} \mu_{\mathrm{i}}$, there exists no dual Lagrange multiplier space $M_{h}\left(\gamma_{k}\right)$ such that $W_{h}^{1}\left(\gamma_{k}\right) \subset M_{h}\left(\gamma_{k}\right)$.

Proof. We prove this by contradiction. Assume that

$$
\sum_{i} \alpha_{i} \mu_{i}=\phi_{p}^{l}
$$

where $\phi_{p}^{l}$ is the bilinear hat function associated with the interior vertex $p$ having the coordinates $(0,0)$, see Figure 3. Suppose the coordinates of the four corners of the face $F_{1}$ be $(-1,0),(0,0),(0,1)$ and $(-1,1)$, and of the face $F_{2}$ be $(0,0),(1,0),(1,1)$ and $(0,1)$.

Because of the duality, the functions $\mu_{i}$ are biorthogonal to the finite element basis functions $\varphi_{i}$ on the interface. Hence, after multiplying (3.2) by some finite element basis function $\varphi_{j}$ and integrating over the interface $\gamma_{k}$, we get

$$
\alpha_{j}=\frac{\int_{\gamma_{k}} \varphi_{j} \phi_{p}^{l} \mathrm{~d} \sigma}{\int_{\gamma_{k}} \varphi_{j} \mathrm{~d} \sigma}
$$

Let $j_{0}$ be the interior vertex with coordinates $(0,1)$ such that $j_{0}$ and $p$ share one edge, see Figure 3 . Then, we find

$$
\int_{\gamma_{k}} \varphi_{j_{0}} \phi_{p}^{l} \mathrm{~d} \sigma=\int_{T_{1}} \varphi_{j_{0}} \phi_{p}^{l} \mathrm{~d} \sigma+\int_{T_{2}} \varphi_{j_{0}} \phi_{p}^{l} \mathrm{~d} \sigma=-\frac{1}{18},
$$

and thus $\alpha_{j_{0}} \neq 0$. Since the basis functions $\mu_{i}$ are locally linearly independent, we obtain $\operatorname{supp} \mu_{\mathrm{j}_{0}} \subseteq \operatorname{supp} \sum_{\mathrm{i}} \alpha_{\mathrm{i}} \mu_{\mathrm{i}}$. By construction, we find $\operatorname{supp} \mu_{\mathrm{j}_{0}} \subsetneq \operatorname{supp} \phi_{\mathrm{p}}^{1}$, which contradicts (3.2).

\subsection{Lagrange multiplier spaces for serendipity elements}

The previous subsection shows that there does not exist a dual Lagrange multiplier space for serendipity elements containing the bilinear hat function at each vertex and satisfying $\operatorname{supp} \varphi_{\mathrm{i}}=\operatorname{supp} \mu_{\mathrm{i}}$. Here, we consider two different Lagrange multiplier spaces for serendipity elements. The essential point is that the Lagrange multiplier space should lead to an optimal and stable discretization scheme. For this purpose, the assumptions [P0]-[P2] are crucial. The first idea is to choose a standard Lagrange multiplier space, see $[5,6]$. In this case, the basis functions for each interior face $F \in S_{k ; h_{k}}$ of the interface $\gamma_{k}$ (i.e., $F \in S_{k ; h_{k}}$ with $\partial F \cap \partial \gamma_{k}=\emptyset$ ) are serendipity basis functions in 2D. All nodes on $\partial \gamma_{k}$ do not carry a degree of freedom for the Lagrange multipliers. Therefore, in order to satisfy assumption [P1], it is necessary to modify the definition of the basis functions for the faces touching the boundary $\partial \gamma_{k}$ of the interface $\gamma_{k}$. Suppose a face $F \in S_{k ; h_{k}}$ with $\partial F \cap \partial \gamma_{k} \neq \emptyset$ has 
$n$ degrees of freedom for the Lagrange multipliers. Then, the local Lagrange multiplier basis function $\mu_{i}$ at a node $x_{i}$ of $F$ is chosen to be a polynomial of minimal degree such that $\mu_{i}\left(x_{j}\right)=\delta_{i j}$ for all $x_{j}, j=1, \cdots, n$. Here, $\delta_{i j}$ is the Kronecker delta. These Lagrange multiplier basis functions are continuous. Working with a continuous Lagrange multiplier space which locally contains the linear functions has the advantage that assumption [P1] is satisfied. Assumption [P0] is trivially satisfied by construction. To verify assumption [P2], we take $\varphi:=\sum_{k=1}^{n_{k}} a_{k} \varphi_{k}$ in $W_{0, h}\left(\gamma_{k}\right)$ and define $\mu:=\sum_{k=1}^{n_{k}} a_{k} \mu_{k}$. Then

$$
(\varphi, \mu)_{0, \gamma_{k}}=\sum_{i, j=1}^{n_{k}} a_{i} a_{j}\left(\varphi_{i}, \mu_{j}\right)_{0, \gamma_{k}}=\sum_{i, j=1}^{n_{k}} a_{i} a_{j} \int_{\gamma_{k}} \varphi_{i} \mu_{j} \mathrm{~d} \sigma .
$$

Computing the local mass matrices on the reference face for the different boundary cases, we find that all eigenvalues of the local mass matrices are greater than $\frac{1}{100}$ and smaller than $\frac{6}{11}$. Then $(\varphi, \mu)_{0, \gamma_{k}},\|\varphi\|_{0, \gamma_{k}}^{2}$ and $\|\mu\|_{0, \gamma_{k}}^{2}$ are equivalent to $\sum_{i=1}^{n_{k}} h_{s(k)}^{2} a_{i}^{2}$, which guarantees assumption [P2]. The coupling of the local mass matrices yields a global mass matrix which is sparse but has a band structure of band-width $O(1 / h)$. Thus, the inverse of the global mass matrix $M_{s}$ on the slave side is dense. As a consequence, we obtain a stiffness matrix associated with the variational problem (1.3), which is not sparse. Then we cannot apply static condensation, and the multigrid method discussed in [22] cannot be used.

To overcome this difficulty, we generalize the concept of dual Lagrange multipliers. The idea is to use a Lagrange multiplier space which yields a sparse inverse of the global mass matrix $M_{s}$ on the slave side. Such a Lagrange multiplier space will be called a quasi-dual Lagrange multiplier space. Working with the tensor product dual basis functions associated with the degrees of freedom of serendipity elements yields a diagonal mass matrix $M_{s}$ and the conditions [P0] and [P2] are satisfied. However, [P1] is not satisfied, and although the discretization scheme is stable, no optimal a priori bounds can be obtained. Now in a first step, we enrich the Lagrange multiplier space to guarantee [P1]. As a result, condition [P0] is lost and thus the inf-sup condition for the Lagrange multiplier space. Therefore, we have to augment the trace space in a second step. The second step can be viewed as a stabilization technique and is well known within the framework of three-field approaches, see, e.g., [12], [11] and [13]. This step guarantees that after enriching the Lagrange multiplier space, the conditions $[\mathrm{P} 0]-[\mathrm{P} 2]$ are satisfied. To perform the second step, we enrich each non-empty face $F \subseteq \partial T \cap \Gamma$ of the element $T$ of the slave side with a bubble function. The bubble function $b \in H^{1}(T)$ corresponding to the face $F$ of $T$ has the property that $b_{\left.\right|_{\partial T \backslash F}}=0$ and $\int_{F} b \mathrm{~d} \sigma \neq 0$. We define $\mathcal{K}_{s}:=\left\{T \in \mathcal{T}_{s(k) ; h_{s(k)}}, 1 \leq k \leq N \mid \partial T \cap \Gamma\right.$ contains at least one face of $\left.T\right\}$. Now, the space of bubble functions $B_{h}$ is formed by $N_{s}$ bubbles, where $N_{s}$ is the number of faces in $\cup_{k=1}^{N} \mathcal{S}_{k ; h_{k}}$, and each of them is associated to a face $F$ of an element $T \in \mathcal{K}_{s}$, where $F \subseteq \partial T \cap \Gamma$. This leads to one additional degree of freedom for each non-empty face $F \subseteq \partial T \cap \Gamma$ of $T \in \mathcal{K}_{s}$. There are many possibilities to define such a bubble function. Here, the triquadratic nodal finite element function associated with the center of gravity of the face is used as a bubble function corresponding to this face. Although we need only the restriction of the bubble functions to the associated face to satisfy assumption [P0], each bubble function is supported on the whole element. Now, the modified unconstrained product space $X_{h}^{t}$ can be written as $X_{h}^{t}=X_{h}^{s} \oplus B_{h}$, where $X_{h}^{s}$ is the unconstrained product space associated with serendipity elements. In the sequel, the space $X_{h}^{t}$ will be called augmented serendipity space and the corresponding elements augmented serendipity elements. This leads to a mass matrix $M_{s}$ on the slave side having a special structure. Suppose $\hat{\varphi}_{i}$ and $\hat{\lambda}_{i}, 1 \leq i \leq 9$ be the local basis functions of the standard triquadratic finite elements and their dual Lagrange multipliers, respectively. Here, the first four basis functions correspond to the vertices, the second four ones correspond to the midpoints of the edges, and the last one corresponds to the center of gravity of the reference face $\hat{F}$. Then, the local basis functions of serendipity elements can be written as $\hat{\varphi}_{i}^{s}=\hat{\varphi}_{i}+\alpha_{i} \hat{\varphi}_{9}, 1 \leq i \leq 8$, where $\alpha_{i}=-\frac{1}{4}$ for $1 \leq i \leq 4$ and $\alpha_{i}=\frac{1}{2}$ for $5 \leq i \leq 8$. Using the biorthogonality of $\hat{\varphi}_{i}$ and $\hat{\lambda}_{i}$, we have

$$
\int_{\hat{F}} \hat{\varphi}_{i}^{s} \hat{\lambda}_{j} \mathrm{~d} \sigma=\int_{\hat{F}}\left(\hat{\varphi}_{i}+\alpha_{i} \hat{\varphi}_{9}\right) \hat{\lambda}_{j} \mathrm{~d} \sigma=\delta_{i j} \int_{\hat{F}} \hat{\varphi}_{i} \mathrm{~d} \sigma+\alpha_{i} \delta_{9 j} \int_{\hat{F}} \hat{\varphi}_{9} \mathrm{~d} \sigma .
$$


In fact, the mass matrix on the reference face $\hat{F}$ is

$$
M_{\hat{F}}=\left[\begin{array}{ccccccccc}
\frac{1}{36} & 0 & 0 & 0 & 0 & 0 & 0 & 0 & 0 \\
0 & \frac{1}{36} & 0 & 0 & 0 & 0 & 0 & 0 & 0 \\
0 & 0 & \frac{1}{36} & 0 & 0 & 0 & 0 & 0 & 0 \\
0 & 0 & 0 & \frac{1}{36} & 0 & 0 & 0 & 0 & 0 \\
0 & 0 & 0 & 0 & \frac{1}{9} & 0 & 0 & 0 & 0 \\
0 & 0 & 0 & 0 & 0 & \frac{1}{9} & 0 & 0 & 0 \\
0 & 0 & 0 & 0 & 0 & 0 & \frac{1}{9} & 0 & 0 \\
0 & 0 & 0 & 0 & 0 & 0 & 0 & \frac{1}{9} & 0 \\
-\frac{1}{9} & -\frac{1}{9} & -\frac{1}{9} & -\frac{1}{9} & \frac{2}{9} & \frac{2}{9} & \frac{2}{9} & \frac{2}{9} & \frac{4}{9}
\end{array}\right] .
$$

To show the consequence of our new Lagrange multiplier space, we consider the global mass matrix $M_{s}$ on the slave side in more detail. In the following, we use the same notation for the vector representation of the solution and the solution as an element in $X_{h}^{t}$ and $M_{h}$. The matrix $A$ is the stiffness matrix associated with the bilinear form $a(\cdot, \cdot)$ on $X_{h}^{t} \times X_{h}^{t}$, and the matrices $B$ and $B^{T}$ are associated with the bilinear form $b(\cdot, \cdot)$ on $X_{h}^{t} \times M_{h}$. Then, the algebraic formulation of the saddle point problem (1.4) is given by

$$
\left[\begin{array}{cc}
A & B^{T} \\
B & 0
\end{array}\right]\left[\begin{array}{l}
u_{h} \\
\lambda_{h}
\end{array}\right]=\left[\begin{array}{c}
f_{h} \\
0
\end{array}\right] .
$$

We recall the grouping of the degrees of freedom of $X_{h}^{t}$ introduced in Section 1. After augmenting the serendipity space with the space of bubble functions $B_{h}$, we further decompose the degrees of freedom associated with the interior nodes of $\gamma_{k}, 1 \leq k \leq N$, on the slave side into two groups $\left(u_{s}, u_{b}\right)$. Here, the block vector $u_{s}$ contains all nodal values of $u$ at the interior nodes of $\gamma_{k}, 1 \leq k \leq N$, corresponding to the vertices and edges on the slave side, and $u_{b}$ stands for all nodal values corresponding to the bubble functions on the slave side. With this decomposition, we can write $u_{h}^{T}=\left(u_{i}^{T}, u_{m}^{T}, u_{s}^{T}, u_{b}^{T}\right)$. The block vector $\lambda_{h}$ containing the nodal values of the Lagrange multiplier is similarly decomposed with $\lambda_{h}^{T}=\left(\lambda_{s}^{T}, \lambda_{b}^{T}\right)$. In terms of this decomposition, we can rewrite the algebraic form of the saddle point problem (3.4) as

$$
\left[\begin{array}{cccccc}
A_{i i} & A_{i m} & A_{i s} & A_{i b} & 0 & 0 \\
A_{m i} & A_{m m} & A_{m s} & A_{m b} & M_{m}^{T} & M_{b m}^{T} \\
A_{s i} & A_{s m} & A_{s s} & A_{s b} & D_{s} & M_{b s}^{T} \\
A_{b i} & A_{b m} & A_{b s} & A_{b b} & 0 & D_{b} \\
0 & M_{m} & D_{s} & 0 & 0 & 0 \\
0 & M_{b m} & M_{b s} & D_{b} & 0 & 0
\end{array}\right]\left[\begin{array}{c}
u_{i} \\
u_{m} \\
u_{s} \\
u_{b} \\
\lambda_{s} \\
\lambda_{b}
\end{array}\right]=\left[\begin{array}{c}
f_{i} \\
f_{m} \\
f_{s} \\
f_{b} \\
0 \\
0
\end{array}\right]
$$

Recalling the algebraic structure (1.5) of the bilinear form $b(\cdot, \cdot)$ restricted to $X_{h}^{t} \times M_{h}$, we have

$$
B=\left[\begin{array}{cccc}
0 & M_{m} & D_{s} & 0 \\
0 & M_{b m} & M_{b s} & D_{b}
\end{array}\right],
$$


where $D_{b}$ and $D_{s}$ are diagonal matrices, and $M_{b s}, M_{m}$ and $M_{b m}$ are rectangular matrices. The matrix $D_{b}$ is diagonal due to the fact that the bubble functions are supported only in one face, and the diagonal form of $D_{s}$ follows from the structure of the local mass matrix, see (3.3). Hence, the global mass matrix $M_{s}$ on the slave side and its inverse $M_{s}^{-1}$ can be written as

$$
M_{s}=\left[\begin{array}{cc}
D_{s} & 0 \\
M_{b s} & D_{b}
\end{array}\right] \text { and } M_{s}^{-1}=\left[\begin{array}{cc}
D_{s}^{-1} & 0 \\
-D_{b}^{-1} M_{b s} D_{s}^{-1} & D_{b}^{-1}
\end{array}\right]
$$

The great benefit of this Lagrange multiplier space is that the inverse of the mass matrix $M_{s}$ can be computed very easily, and the inverse is sparse. Thus, the solution on the slave side depends locally on the solution on the master side. Here, we have to invert only two diagonal matrices and scale $M_{b s}$ to compute the inverse of the mass matrix $M_{s}$. The stiffness matrix associated with the variational problem (1.3) is sparse, and efficient iterative solver like multigrid can easily be adapted to the nonconforming situation. Furthermore, the degrees of freedom corresponding to the bubble functions can locally be eliminated by static condensation. Since the matrix $D_{b}$ is diagonal, the sixth and the fourth line of the system (3.5) give

$$
\begin{aligned}
& u_{b}=-D_{b}^{-1}\left(M_{b m} u_{m}+M_{b s} u_{s}\right), \text { and } \\
& \lambda_{b}=D_{b}^{-1}\left[f_{b}-A_{b i} u_{i}-\left(A_{b m}-A_{b b} D_{b}^{-1} M_{b m}\right) u_{m}-\left(A_{b s}-A_{b b} D_{b}^{-1} M_{b s}\right) u_{s}\right] .
\end{aligned}
$$

Now, we eliminate $u_{b}$ and $\lambda_{b}$ from the system (3.5) and obtain a new system

$$
\hat{A} \hat{u}_{h}=\hat{F}_{h}
$$

where $\hat{u}_{h}^{T}=\left(u_{i}^{T}, u_{m}^{T}, u_{s}^{T}, \lambda_{s}^{T}\right)$. Defining $M_{1}:=D_{b}^{-1} M_{b m}$ and $M_{2}:=D_{b}^{-1} M_{b s}$, we have

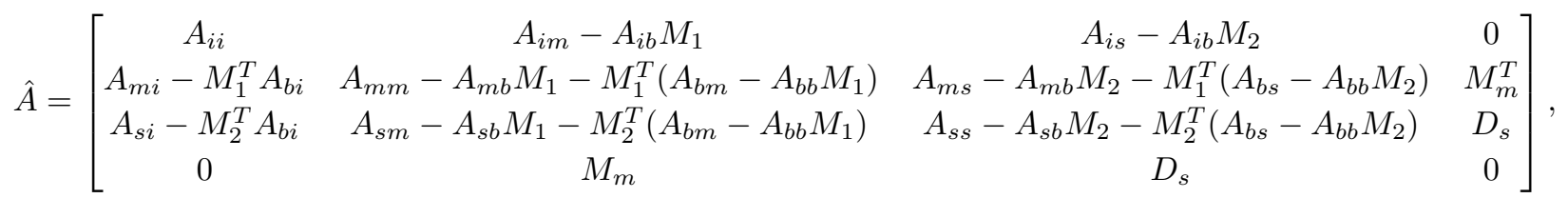

and the right hand side can be written as

$$
\hat{F}_{h}=\left[\begin{array}{c}
f_{i} \\
f_{m}-M_{1}^{T} f_{b} \\
f_{s}-M_{2}^{T} f_{b} \\
0
\end{array}\right]
$$

We observe that the matrix $\hat{A}$ is symmetric, if $A$ is symmetric and it has exactly the same structure as the saddle point matrix arising from mortar finite element method with a dual Lagrange multiplier space, see [21]. Because of this structure of the algebraic system, we can apply the multigrid method proposed in [22].

Remark 3.2. There is also a possibility to use wavelets to get a mass matrix of special structure so that the inversion can be cheaper, and the inverse is sparse. In [18], locally supported and piecewise polynomial wavelets are studied on non-uniform meshes which give a lower triangular mass matrix with higher order finite elements in triangular meshes. 


\section{NUMERICAL RESULTS}

Here, we present some numerical examples in 3D for linear and quadratic mortar finite elements. We consider three different cases for quadratic mortar finite elements. The first one is the standard triquadratic finite element space with the dual Lagrange multiplier space introduced in Subsection 3.1. The second one is the serendipity space with a standard Lagrange multiplier space given in Subsection 3.2. Finally, the third one is the augmented serendipity space associated with the tensor product Lagrange multiplier space, which is a quasi-dual Lagrange multiplier space. Our numerical results show the same asymptotic behavior as predicted by the theory. The implementation is based on the finite element toolbox ug, [1]. We do not discuss and analyze an iterative solver for the arising linear systems. Working with dual or quasi-dual Lagrange multiplier spaces has the advantage that the flux can locally be eliminated, and static condensation yields a positive definite system on the unconstrained product space. In [21,22], the modification of the system has been carried out and a local modification of the transfer operators of lower complexity has been proposed. The introduced multigrid has a level-independent convergence rate and is of optimal complexity. Unfortunately, in the case of a standard Lagrange multiplier space no local elimination of the flux can be carried out. Following the approach in [22], the sparsity of the modified system and the efficiency of the multigrid solver is lost. In that case, we apply a multigrid method for saddle point problems. This technique has been considered for mortar elements in [19] and further analyzed in $[8,20]$. It turns out that we do not have to work in a positive definite subspace, and the smoother can be realized by an inner and outer iteration scheme. As in the other approach, level-independent multigrid convergence rates can be established. However, the numerical solution process is slower if we have to work with the saddle point approach. We point out that the more efficient multigrid method for the modified positive definite system can only be applied when the inverse of $M_{s}$ is sparse, whereas the saddle point multigrid method is more general. We present some numerical results in 3D illustrating the performance of the different Lagrange multiplier spaces. In particular, we compare the discretization errors in the $L^{2}$ - and $H^{1}$ - norm for the solution for linear and quadratic mortar finite elements. The discretization errors in the flux across the interface are compared in a mesh-dependent Lagrange multiplier norm, which is defined by

$$
\left\|\mu-\mu_{h}\right\|_{h}^{2}:=\sum_{m=1}^{N} \sum_{F \in \mathcal{S}_{m ; h_{m}}} h_{F}\left\|\mu-\mu_{h}\right\|_{0, F}^{2},
$$

where $h_{F}$ is the diameter of the face $F$. For all our examples, we have used uniform refinement. In each refinement step, the elements are refined into eight subelements. We denote by $X_{h}^{l}$ and $X_{h}^{f}$ the unconstrained finite element spaces associated with the standard finite element spaces for the trilinear and the triquadratic case, respectively. Similarly, $X_{h}^{s}$ and $X_{h}^{t}$ are the unconstrained finite element spaces associated with the serendipity elements and the augmented serendipity elements as defined in the previous section, respectively. The corresponding finite element solutions are denoted by $u_{h}^{l}, u_{h}^{f}, u_{h}^{s}$ and $u_{h}^{t}$, respectively.

Remark 4.1. We note that the concept of dual Lagrange multiplier spaces can be generalized to distorted hexahedral meshes. In that case, the mapping between the actual element and the reference element has a non-constant Jacobian. As a consequence, we have to compute for each face on the interface a biorthogonal basis with respect to the local nodal one. This can be easily done by solving a local mass matrix system. By construction, the sum of the local dual Lagrange multiplier basis functions is one. Defining the global Lagrange multiplier basis functions by gluing the local ones together, we find that the constants are included in the Lagrange multiplier space. As a consequence, it is easy to verify that the discretization error is of order $h$ for lowest order finite elements.

In our Example 1, we choose a L-shaped domain. The domain $\Omega:=\left((0,1)^{2} \times(0,2)\right) \cup\left([1,2) \times(0,1)^{2}\right)$ is decomposed into three cubes, $\Omega_{1}:=(0,1)^{3}, \Omega_{2}:=(0,1)^{2} \times(1,2)$ and $\Omega_{3}:=(1,2) \times(0,1)^{2}$. We have shown the decomposition of the domain and the initial triangulation in the left picture of Figure 4 , and the isolines of the solution at the interface $z=1$ are shown in the right. Here, we solve a Poisson problem $-\Delta u=f$ 

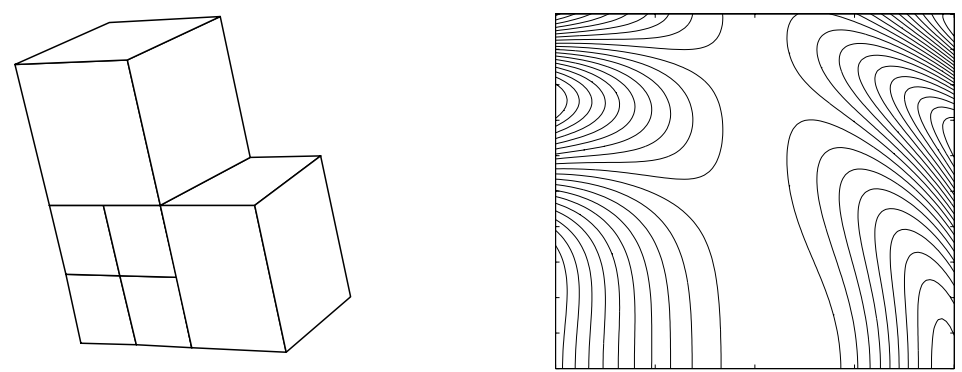

Figure 4. Decomposition of the domain and initial triangulation (left), isolines of the solution at the interface $z=1$ (right), Example 1 .

TABLE 1. Discretization errors in the $L^{2}$-norm, Example 1.

\begin{tabular}{|c|c|c|c|c|c|}
\hline level & $\#$ elem. & $\left\|u-u_{h}^{l}\right\|_{0}$ & $\left\|u-u_{h}^{f}\right\|_{0}$ & $\left\|u-u_{h}^{s}\right\|_{0}$ & $\left\|u-u_{h}^{t}\right\|_{0}$ \\
\hline 0 & 10 & $1.327466 \mathrm{e}+00$ & $7.318159 \mathrm{e}-01$ & $8.066003 \mathrm{e}-01$ & $7.957931 \mathrm{e}-01$ \\
\hline 1 & 80 & $8.047675 \mathrm{e}-01$ & $1.748627 \mathrm{e}-01$ & $2.039559 \mathrm{e}-01$ & $2.008262 \mathrm{e}-01$ \\
\hline 2 & 640 & $2.057468 \mathrm{e}-01$ & $4.863715 \mathrm{e}-02$ & $4.936495 \mathrm{e}-02$ & $4.910636 \mathrm{e}-02$ \\
\hline 3 & 5120 & $6.722455 \mathrm{e}-02$ & $6.422065 \mathrm{e}-03$ & $6.451969 \mathrm{e}-03$ & $6.443622 \mathrm{e}-03$ \\
\hline 4 & 40960 & $1.766195 \mathrm{e}-02$ & $8.056078 \mathrm{e}-04$ & $8.064556 \mathrm{e}-04$ & $8.066527 \mathrm{e}-04$ \\
\hline
\end{tabular}

TABLE 2. Discretization errors in the $H^{1}$-norm, Example 1.

\begin{tabular}{|c|c|c|c|c|c|}
\hline level & $\#$ elem. & $\left\|u-u_{h}^{l}\right\|_{1}$ & $\left\|u-u_{h}^{f}\right\|_{1}$ & $\left\|u-u_{h}^{s}\right\|_{1}$ & $\left\|u-u_{h}^{t}\right\|_{1}$ \\
\hline 0 & 10 & $1.021784 \mathrm{e}+00$ & $8.085453 \mathrm{e}-01$ & $8.194130 \mathrm{e}-01$ & $8.049068 \mathrm{e}-01$ \\
\hline 1 & 80 & $8.094756 \mathrm{e}-01$ & $3.934127 \mathrm{e}-01$ & $4.259780 \mathrm{e}-01$ & $4.159907 \mathrm{e}-01$ \\
\hline 2 & 640 & $4.221967 \mathrm{e}-01$ & $1.803404 \mathrm{e}-01$ & $1.831986 \mathrm{e}-01$ & $1.814987 \mathrm{e}-01$ \\
\hline 3 & 5120 & $2.479979 \mathrm{e}-01$ & $4.695275 \mathrm{e}-02$ & $4.711321 \mathrm{e}-02$ & $4.701404 \mathrm{e}-02$ \\
\hline 4 & 40960 & $1.277419 \mathrm{e}-01$ & $1.173059 \mathrm{e}-02$ & $1.173325 \mathrm{e}-02$ & $1.173569 \mathrm{e}-02$ \\
\hline
\end{tabular}

TABLE 3. Discretization errors in the weighted Lagrange multiplier norm, Example 1.

\begin{tabular}{|c|c|c|c|c|c|}
\hline level & $\#$ elem. & $\left\|\lambda-\lambda_{h}^{l}\right\|_{h}$ & $\left\|\lambda-\lambda_{h}^{f}\right\|_{h}$ & $\left\|\lambda-\lambda_{h}^{s}\right\|_{h}$ & $\left\|\lambda-\lambda_{h}^{t}\right\|_{h}$ \\
\hline 0 & 10 & $9.992731 \mathrm{e}-01$ & $5.988951 \mathrm{e}+00$ & $4.678395 \mathrm{e}+00$ & $7.046350 \mathrm{e}+00$ \\
\hline 1 & 80 & $2.416457 \mathrm{e}+00$ & $1.831235 \mathrm{e}+00$ & $3.100680 \mathrm{e}+00$ & $2.018278 \mathrm{e}+00$ \\
\hline 2 & 640 & $8.795363 \mathrm{e}-01$ & $5.338894 \mathrm{e}-01$ & $7.909840 \mathrm{e}-01$ & $6.360541 \mathrm{e}-01$ \\
\hline 3 & 5120 & $4.481377 \mathrm{e}-01$ & $7.548844 \mathrm{e}-02$ & $9.720802 \mathrm{e}-02$ & $1.002547 \mathrm{e}-01$ \\
\hline 4 & 40960 & $1.720963 \mathrm{e}-01$ & $1.157310 \mathrm{e}-02$ & $1.577079 \mathrm{e}-02$ & $1.684356 \mathrm{e}-02$ \\
\hline
\end{tabular}

with the right hand side function $f$ and the Dirichlet boundary conditions determined by the exact solution $u(x, y, z)=\left((x-1)^{2}+(z-1)^{2}\right)^{5 / 6} \cos \left(6 y^{2}+x^{2}+6\right)$.

We have tabulated the discretization errors in different norms in Tables $1-3$. Here, the solution is not $H^{3}-$ regular. Since the solution $u \in H^{8 / 3-\epsilon}(\Omega)$ for $\epsilon>0$, we expect the convergence of order one in the $H^{1}$-norm for the linear case. In the quadratic case, we cannot expect a convergence of order two in this norm. In all three cases of quadratic finite elements, we observe asymptotic rates in the $L^{2}$ - and $H^{1}$-norm, which are better than 

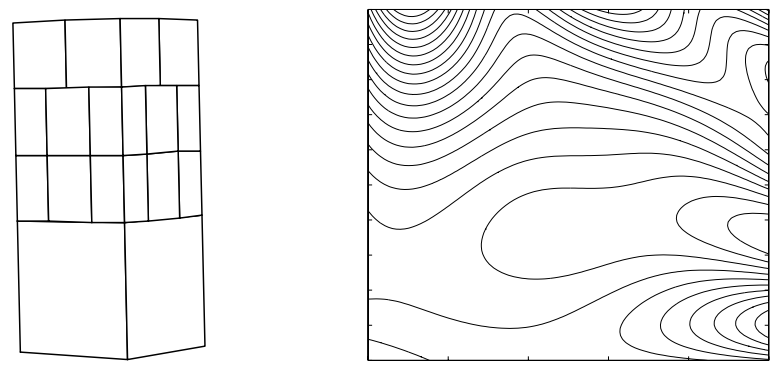

Figure 5. Decomposition of the domain and initial triangulation (left), isolines of the solution at the interface $z=2$ (right), Example 2 .

TABLE 4. Discretization errors in the $L^{2}$-norm, Example 2.

\begin{tabular}{|c|c|c|c|c|c|c|c|}
\hline level & \# elem. & \multicolumn{1}{|c|}{$\left\|u-u_{h}^{l}\right\|_{0}$} & \multicolumn{2}{|c|}{$\left\|u-u_{h}^{s}\right\|_{0}$} & \multicolumn{2}{|c|}{$\left\|u-u_{h}^{t}\right\|_{0}$} \\
\hline 0 & 23 & $8.911337 \mathrm{e}-01$ & & $1.745670 \mathrm{e}-01$ & & $1.760480 \mathrm{e}-01$ & 0 \\
\hline 1 & 184 & $2.582954 \mathrm{e}-01$ & 1.79 & $2.997997 \mathrm{e}-02$ & 2.54 & $3.010899 \mathrm{e}-02$ & 2.55 \\
\hline 2 & 1472 & $6.366337 \mathrm{e}-02$ & 2.02 & $3.664595 \mathrm{e}-03$ & 3.03 & $3.671731 \mathrm{e}-03$ & 3.04 \\
\hline 3 & 11776 & $1.607229 \mathrm{e}-02$ & 1.99 & $4.466631 \mathrm{e}-04$ & 3.04 & $4.462098 \mathrm{e}-04$ & 3.04 \\
\hline 4 & 94208 & $4.031862 \mathrm{e}-03$ & 2.00 & $5.393667 \mathrm{e}-05$ & 3.05 & $5.391429 \mathrm{e}-05$ & 3.05 \\
\hline
\end{tabular}

predicted by the theory. The quantitative results are almost the same in these norms. Theoretically, the errors in the weighted Lagrange multiplier norm for the quadratic and linear case are expected to be of order $h^{2}$ and $h$ in the optimal case, respectively. Here, we observe better rates of convergence for the errors in the weighted Lagrange multiplier norm. The better convergence rates are due to the fact that the error in the $H^{1}$-norm is equally distributed and the Lagrange multiplier space has an $O\left(h^{5 / 2}\right)$ and $O\left(h^{3 / 2}\right)$ approximation property in the considered norm.

In Example 1, there is not any significant difference in the accuracy between the different quadratic mortar solutions neither in the $L^{2}$-norm nor in the $H^{1}$-norm. However, a quantitative difference can be seen for the discretization errors in the weighted Lagrange multiplier norm. In this norm, the standard triquadratic finite elements with the tensor product Lagrange multiplier space gives the best results, whereas the difference between the augmented serendipity elements with the quasi-dual Lagrange multiplier space and the serendipity elements with the standard Lagrange multiplier space is quite negligible.

For the next three examples, we consider only linear and serendipity elements. In our second example, the domain $\Omega:=(0,1)^{2} \times(0,2.5)$ is decomposed into three subdomains $\Omega_{1}:=(0,1)^{3}, \Omega_{2}:=(0,1)^{2} \times(1,2)$, and $\Omega_{3}:=(0,1)^{2} \times(2,2.5)$. The right hand side $f$ and the boundary conditions of $-\Delta u=f$ are chosen such that the exact solution is given by $u(x, y, z)=5(z-1.4)\left((x-0.5)^{2}+4(y-0.3)^{3}\right)+z(z-1) \sin (4 \pi x y)\left(2(x-y)^{2}+(y+x-1)^{2}\right)$. In Figure 5, we have shown the decomposition of the domain, the initial nonmatching triangulation and the isolines of the solution at the interface $z=2$. Here, we have three subdomains and two interfaces. The middle cube is taken as the slave side. We start with a nonconforming coarse initial triangulation having 23 elements. The discretization errors along with their order of convergence at every refinement step in different norms are given in Tables 4-6. As before, we get the correct asymptotic rates for both cases of serendipity elements. The errors in the $L^{2}$ - and $H^{1}$-norm are almost the same for both approaches. In the weighted Lagrange multiplier norm, the serendipity elements yield smaller errors than the augmented serendipity elements. However, the difference is quite negligible, and the asymptotic rate of convergence is optimal in both cases. In our third example, we consider a domain $\Omega:=(0,2) \times(0,1) \times(0,2)$, which is decomposed into four subdomains 
TABLE 5. Discretization errors in the $H^{1}$-norm, Example 2.

\begin{tabular}{|c|c|c|c|c|c|c|c|}
\hline level & \# elem. & \multicolumn{2}{|c|}{$\left\|u-u_{h}^{l}\right\|_{1}$} & \multicolumn{2}{|c|}{$\left\|u-u_{h}^{s}\right\|_{1}$} & \multicolumn{2}{|c|}{$\left\|u-u_{h}^{t}\right\|_{1}$} \\
\hline 0 & 23 & $8.170532 \mathrm{e}-01$ & & $5.517577 \mathrm{e}-01$ & & $5.290887 \mathrm{e}-01$ & 0 \\
\hline 1 & 184 & $5.643329 \mathrm{e}-01$ & 0.53 & $1.478160 \mathrm{e}-01$ & 1.90 & $1.482833 \mathrm{e}-01$ & 1.84 \\
\hline 2 & 1472 & $2.626420 \mathrm{e}-01$ & 1.10 & $3.915936 \mathrm{e}-02$ & 1.92 & $3.920488 \mathrm{e}-02$ & 1.92 \\
\hline 3 & 11776 & $1.293053 \mathrm{e}-01$ & 1.02 & $9.352708 \mathrm{e}-03$ & 2.07 & $9.332480 \mathrm{e}-03$ & 2.07 \\
\hline 4 & 94208 & $6.446694 \mathrm{e}-02$ & 1.00 & $2.295583 \mathrm{e}-03$ & 2.03 & $2.293897 \mathrm{e}-03$ & 2.02 \\
\hline
\end{tabular}

TABLE 6. Discretization errors in the weighted Lagrange multiplier norm, Example 2.

\begin{tabular}{|c|c|c|c|c|c|c|c|}
\hline level & \# elem. & \multicolumn{2}{|c|}{$\left\|\lambda-\lambda_{h}^{l}\right\|_{h}$} & \multicolumn{2}{|c|}{$\left\|\lambda-\lambda_{h}^{s}\right\|_{h}$} & \multicolumn{2}{c|}{$\left\|\lambda-\lambda_{h}^{t}\right\|_{h}$} \\
\hline 0 & 23 & $7.433164 \mathrm{e}+00$ & & $2.762317 \mathrm{e}+01$ & & $2.758347 \mathrm{e}+01$ & 0 \\
\hline 1 & 184 & $5.657720 \mathrm{e}+00$ & 0.39 & $2.006842 \mathrm{e}+00$ & 3.78 & $3.320707 \mathrm{e}+00$ & 3.05 \\
\hline 2 & 1472 & $1.855735 \mathrm{e}+00$ & 1.61 & $7.048806 \mathrm{e}-01$ & 1.51 & $8.042462 \mathrm{e}-01$ & 2.05 \\
\hline 3 & 11776 & $4.868778 \mathrm{e}-01$ & 1.93 & $1.001359 \mathrm{e}-01$ & 2.82 & $1.151919 \mathrm{e}-01$ & 2.80 \\
\hline 4 & 94208 & $1.832775 \mathrm{e}-01$ & 1.41 & $1.564914 \mathrm{e}-02$ & 2.68 & $1.879805 \mathrm{e}-02$ & 2.62 \\
\hline
\end{tabular}

$\Omega_{1}:=(0,1)^{3}, \Omega_{2}:=(0,1)^{2} \times(1,2), \Omega_{3}:=(1,2) \times(0,1)^{2}$ and $\Omega_{4}:=(1,2) \times(0,1) \times(1,2)$. We have shown the decomposition of the domain and the initial triangulation in the left picture of Figure 6 , the isolines of the solution on the plane $y=\frac{1}{2}$ in the middle, and the flux of the exact solution at the interface $x=1$ is shown in the right one. Here, $\Omega_{2}$ and $\Omega_{3}$ are taken to be the slave sides and the rest are master sides. In this example, we have one interior macro-edge on which the initial triangulations are non-matching. The problem for this example is given by a reaction-diffusion equation

$$
-\operatorname{div}(a \nabla u)+u=f \quad \text { in } \quad \Omega,
$$

where $a$ is chosen to be 1 in $\Omega_{1}$ and $\Omega_{4}$, and $a=10$ in $\Omega_{2}$ and $\Omega_{3}$. We have chosen the exact solution $u(x, y, z)=(x-1) y(z-1) \exp \left(-(x-1)^{2}-y^{2}-(z-1)^{2}\right) \cos (2 x+2 y+2 z) / a$ and the right hand side $f$ and the Dirichlet boundary conditions are determined from the exact solution. We remark that the exact solution $u$ has a jump in the normal derivative across the interface, whereas the flux is continuous. We have given the discretization errors together with their order of convergence in each refinement step in Tables 7-9. As in the other examples, we get the same asymptotic rates for the $L^{2}$ - and $H^{1}$-norm and better convergence rates in the weighted Lagrange multiplier norm. In contrast to the other examples, we observe numerically a higher convergence order in the weighted Lagrange multiplier norm.

In our last example, we have used a U-shaped domain $\Omega$ decomposed into five subdomains $\Omega_{k}, k=1, \cdots, 5$, and the problem is given by a Poisson equation $-\Delta u=f$. Here, $\Omega_{1}:=(0,1)^{3}, \Omega_{2}:=(0,1)^{2} \times(1,2.4), \Omega_{4}:=$ $(2,3) \times(-0.2,1.2) \times(0,1), \Omega_{5}:=(2,3) \times(-0.2,1.2) \times(1,2)$, and $\Omega_{3}$ is a hexahedral pyramidal frustum joining the domain $\Omega_{1}$ and $\Omega_{4}$, see the left picture of Figure 7 . Here, we choose the right hand side function $f$ and Dirichlet boundary condition on $\partial \Omega$ so that we obtain the exact solution

$$
u(x, y, z)=\exp \left(-\frac{1}{4}\left(x^{2}+y^{2}+z^{2}\right)\right)(\cos (5 x+z)+3 \sin (4 y+z)) .
$$

The isolines of the solution at the plane $z=1$ are given in the right picture of Figure 7 . We have given the discretization errors in different norms in Tables 10-12. As before, we get optimal convergence rates in the $L^{2}$ - and $H^{1}$-norms for both quadratic approaches and better convergence behavior in the weighted Lagrange multiplier norm. 

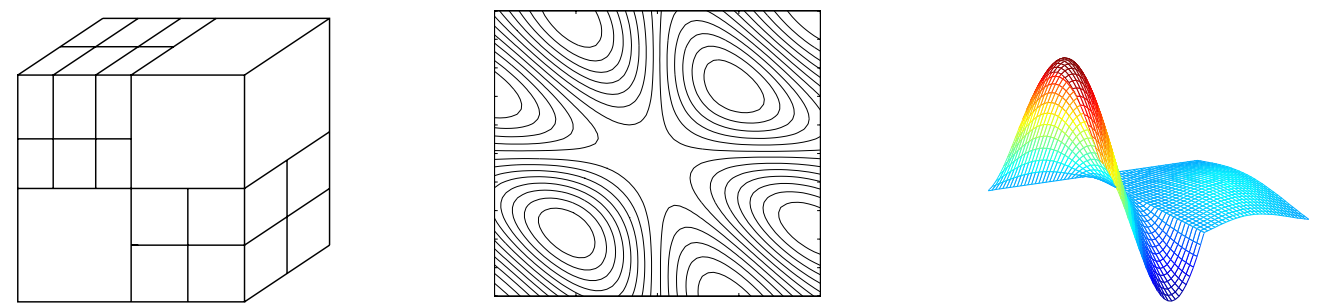

Figure 6. Decomposition of the domain and initial triangulation (left), isolines of the solution at the plane $y=\frac{1}{2}$ (middle) and flux of the exact solution at the interface $x=1$ (right), Example 3.

TABLE 7. Discretization errors in the $L^{2}$-norm, Example 3.

\begin{tabular}{|c|c|c|c|c|c|c|c|}
\hline level & \# elem. & \multicolumn{2}{|l|}{$\left\|u-u_{h}^{l}\right\|_{0}$} & \multicolumn{2}{|l|}{$\left\|u-u_{h}^{s}\right\|_{0}$} & \multicolumn{2}{|l|}{$\left\|u-u_{h}^{t}\right\|_{0}$} \\
\hline 0 & 22 & $4.636300 \mathrm{e}-01$ & & $1.237718 \mathrm{e}-01$ & & $1.233229 \mathrm{e}-01$ & \\
\hline 1 & 176 & $1.218875 \mathrm{e}-01$ & 1.93 & $1.220035 \mathrm{e}-02$ & 3.34 & $1.220072 \mathrm{e}-02$ & 3.34 \\
\hline 2 & 1408 & $3.082112 \mathrm{e}-02$ & 1.98 & $1.164306 \mathrm{e}-03$ & 3.39 & $1.164276 \mathrm{e}-03$ & 3.39 \\
\hline 3 & 11264 & 7.712933e-03 & 2.00 & $1.422899 \mathrm{e}-04$ & 3.03 & $1.422876 \mathrm{e}-04$ & 3.03 \\
\hline 4 & 90112 & $1.928288 \mathrm{e}-03$ & 2.00 & $1.773015 \mathrm{e}-05$ & 3.00 & $1.773010 \mathrm{e}-05$ & 3.00 \\
\hline
\end{tabular}

TABLE 8. Discretization errors in the $H^{1}$-norm, Example 3.

\begin{tabular}{|c|c|c|c|c|c|c|c|}
\hline level & \# elem. & \multicolumn{1}{|c|}{$\left\|u-u_{h}^{l}\right\|_{1}$} & $\left\|u-u_{h}^{s}\right\|_{1}$ & \multicolumn{2}{|c|}{$\left\|u-u_{h}^{t}\right\|_{1}$} \\
\hline 0 & 22 & $6.295650 \mathrm{e}-01$ & & $2.218643 \mathrm{e}-01$ & & $2.205473 \mathrm{e}-01$ & \\
\hline 1 & 176 & $3.009651 \mathrm{e}-01$ & 1.06 & $4.609256 \mathrm{e}-02$ & 2.27 & $4.607911 \mathrm{e}-02$ & 2.26 \\
\hline 2 & 1408 & $1.482825 \mathrm{e}-01$ & 1.02 & $1.029429 \mathrm{e}-02$ & 2.16 & $1.029336 \mathrm{e}-02$ & 2.16 \\
\hline 3 & 11264 & $7.379459 \mathrm{e}-02$ & 1.01 & $2.523696 \mathrm{e}-03$ & 2.03 & $2.523642 \mathrm{e}-03$ & 2.03 \\
\hline 4 & 90112 & $3.684966 \mathrm{e}-02$ & 1.00 & $6.289388 \mathrm{e}-04$ & 2.00 & $6.289362 \mathrm{e}-04$ & 2.00 \\
\hline
\end{tabular}

TABLE 9. Discretization errors in the weighted Lagrange multiplier norm, Example 3.

\begin{tabular}{|c|c|c|c|c|c|c|c|}
\hline level & \# elem. & \multicolumn{1}{|c|}{$\left\|\lambda-\lambda_{h}^{l}\right\|_{h}$} & \multicolumn{2}{|c|}{$\left\|\lambda-\lambda_{h}^{s}\right\|_{h}$} & \multicolumn{2}{|c|}{$\left\|\lambda-\lambda_{h}^{t}\right\|_{h}$} \\
\hline 0 & 22 & $8.588035 \mathrm{e}-02$ & & $6.184646 \mathrm{e}-02$ & & $8.310643 \mathrm{e}-02$ & \\
\hline 1 & 176 & $5.194191 \mathrm{e}-02$ & 0.73 & $5.220039 \mathrm{e}-03$ & 3.57 & $9.849865 \mathrm{e}-03$ & 3.08 \\
\hline 2 & 1408 & $2.538350 \mathrm{e}-02$ & 1.03 & $4.361169 \mathrm{e}-04$ & 3.58 & $7.810953 \mathrm{e}-04$ & 3.66 \\
\hline 3 & 11264 & $1.012701 \mathrm{e}-02$ & 1.33 & $4.506948 \mathrm{e}-05$ & 3.27 & $6.918887 \mathrm{e}-05$ & 3.50 \\
\hline 4 & 90112 & $3.755812 \mathrm{e}-03$ & 1.43 & $5.074655 \mathrm{e}-06$ & 3.15 & $6.546292 \mathrm{e}-06$ & 3.40 \\
\hline
\end{tabular}

In all our examples, we observe optimal asymptotic convergence rates as predicted by the theory. Although we see the same qualitative behavior, some quantitative differences can be observed in the weighted Lagrange multiplier norm. In this norm, the serendipity elements with the standard Lagrange multiplier space gives better results. However, there is not any essential difference in the discretization errors between different quadratic mortar solutions. Since we enrich the skeleton $\Gamma$ by bubble functions from the slave side, $X_{h}^{t}$ has more degree of freedom than $X_{h}^{s}$. However, these bubble functions can locally be eliminated from the algebraic formulation of the saddle point problem leading to a system matrix, which is similar to the algebraic form of the saddle point problem arising from the mortar discretization with a dual Lagrange multiplier space. 

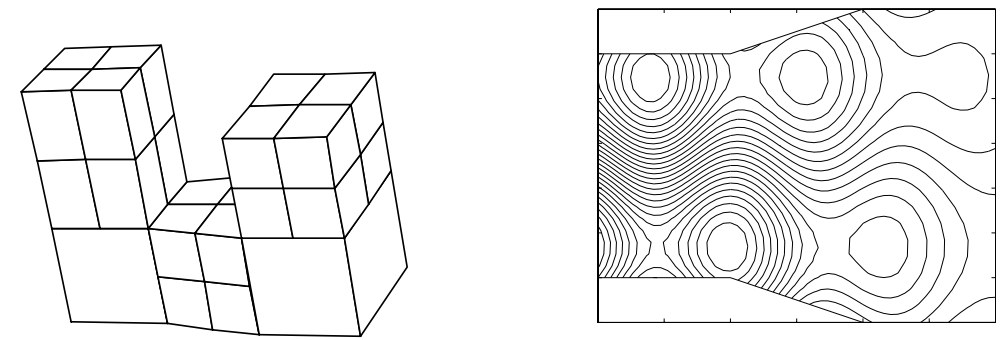

Figure 7. Decomposition of the domain and initial triangulation (left) and isolines of the solution at the plane $z=1$ (right), Example 4 .

TABLE 10. Discretization errors in the $L^{2}$-norm, Example 4.

\begin{tabular}{|c|c|c|c|c|c|c|c|}
\hline level & $\#$ elem. & \multicolumn{2}{|c|}{$\left\|u-u_{h}^{l}\right\|_{0}$} & \multicolumn{2}{|c|}{$\left\|u-u_{h}^{s}\right\|_{0}$} & \multicolumn{2}{|c|}{$\left\|u-u_{h}^{t}\right\|_{0}$} \\
\hline 0 & 26 & $7.310111 \mathrm{e}-01$ & & $3.478550 \mathrm{e}-01$ & & $3.166037 \mathrm{e}-01$ & \\
\hline 1 & 208 & $3.398657 \mathrm{e}-01$ & 1.10 & $4.304959 \mathrm{e}-02$ & 3.01 & $4.209598 \mathrm{e}-02$ & 2.91 \\
\hline 2 & 1664 & $8.175747 \mathrm{e}-02$ & 2.06 & $5.991830 \mathrm{e}-03$ & 2.84 & $5.949794 \mathrm{e}-03$ & 2.82 \\
\hline 3 & 13312 & $2.027467 \mathrm{e}-02$ & 2.01 & $7.579318 \mathrm{e}-04$ & 2.98 & $7.564277 \mathrm{e}-04$ & 2.98 \\
\hline 4 & 106496 & $5.037341 \mathrm{e}-03$ & 2.01 & $9.456858 \mathrm{e}-05$ & 3.00 & $9.451905 \mathrm{e}-05$ & 3.00 \\
\hline
\end{tabular}

TABLE 11. Discretization errors in the $H^{1}$-norm, Example 4.

\begin{tabular}{|c|c|c|c|c|c|c|c|}
\hline level & \# elem. & \multicolumn{1}{|c|}{$\left\|u-u_{h}^{l}\right\|_{1}$} & \multicolumn{2}{|c|}{$\left\|u-u_{h}^{s}\right\|_{1}$} & \multicolumn{2}{|c|}{$\left\|u-u_{h}^{t}\right\|_{1}$} \\
\hline 0 & 26 & $8.982209 \mathrm{e}-01$ & & $5.699839 \mathrm{e}-01$ & & $4.440725 \mathrm{e}-01$ & \\
\hline 1 & 208 & $5.597470 \mathrm{e}-01$ & 0.68 & $1.391079 \mathrm{e}-01$ & 2.03 & $1.308973 \mathrm{e}-01$ & 1.76 \\
\hline 2 & 1664 & $2.683965 \mathrm{e}-01$ & 1.06 & $3.643727 \mathrm{e}-02$ & 1.93 & $3.576211 \mathrm{e}-02$ & 1.87 \\
\hline 3 & 13312 & $1.327426 \mathrm{e}-01$ & 1.02 & $9.024865 \mathrm{e}-03$ & 2.01 & $8.980617 \mathrm{e}-03$ & 1.99 \\
\hline 4 & 106496 & $6.597358 \mathrm{e}-02$ & 1.01 & $2.239350 \mathrm{e}-03$ & 2.01 & $2.236550 \mathrm{e}-03$ & 2.01 \\
\hline
\end{tabular}

TABLE 12. Discretization errors in the weighted Lagrange multiplier norm, Example 4.

\begin{tabular}{|c|c|c|c|c|c|c|c|}
\hline level & \# elem. & \multicolumn{2}{|c|}{$\left\|\lambda-\lambda_{h}^{l}\right\|_{h}$} & \multicolumn{2}{|c|}{$\left\|\lambda-\lambda_{h}^{s}\right\|_{h}$} & \multicolumn{2}{c|}{$\left\|\lambda-\lambda_{h}^{t}\right\|_{h}$} \\
\hline 0 & 26 & $8.792140 \mathrm{e}+00$ & & $1.351765 \mathrm{e}+01$ & & $1.416514 \mathrm{e}+01$ & \\
\hline 1 & 208 & $5.592290 \mathrm{e}+00$ & 0.65 & $2.113658 \mathrm{e}+00$ & 2.68 & $1.567016 \mathrm{e}+00$ & 3.18 \\
\hline 2 & 1664 & $1.963516 \mathrm{e}+00$ & 1.51 & $3.166530 \mathrm{e}-01$ & 2.74 & $3.365031 \mathrm{e}-01$ & 2.22 \\
\hline 3 & 13312 & $7.490561 \mathrm{e}-01$ & 1.39 & $4.610005 \mathrm{e}-02$ & 2.78 & $5.699660 \mathrm{e}-02$ & 2.56 \\
\hline 4 & 106496 & $2.745105 \mathrm{e}-01$ & 1.45 & $7.388080 \mathrm{e}-03$ & 2.64 & $9.779245 \mathrm{e}-03$ & 2.54 \\
\hline
\end{tabular}

Furthermore, the growth rate of the number of bubble functions is only a factor of four in each refinement step, and restricted to the skeleton. This is negligible since we can work with an efficient multigrid solver in case of the augmented serendipity space with the quasi-dual Lagrange multiplier space. Although we can work with the efficient multigrid solver in case of standard triquadratic finite elements, the approach is not as optimal as the augmented serendipity approach due to the higher number of degrees of freedom. It turns out that the most efficient approach is the one given by the augmented serendipity elements. The discretization errors are as good as in the other cases, and the numerical solution is cheaper. 


\section{REFERENCES}

[1] P. Bastian, K. Birken, K. Johannsen, S. Lang, N. Neuß, H. Rentz-Reichert and C. Wieners, UG - a flexible software toolbox for solving partial differential equations. Comput. Vis. Sci. 1 (1997) 27-40.

[2] F. Ben Belgacem, The mortar finite element method with Lagrange multipliers. Numer. Math. 84 (1999) 173-197.

[3] F. Ben Belgacem and Y. Maday, The mortar element method for three dimensional finite elements. RAIRO Modél. Math. Anal. Numér. 31 (1997) 289-302.

[4] C. Bernardi, N. Debit and Y. Maday, Coupling finite element and spectral methods: First results. Math. Comp. 54 (1990) 21-39.

[5] C. Bernardi, Y. Maday and A.T. Patera, Domain decomposition by the mortar element method, in Asymptotic and numerical methods for partial differential equations with critical parameters, H.G. Kaper and M. Garbey Eds., NATO ASI Series 39 (1993) 269-286.

[6] C. Bernardi, Y. Maday and A.T. Patera, A new nonconforming approach to domain decomposition: the mortar element method, in Nonlinear partial differential equations and their applications, H. Brezzi and J.-L. Lions Eds., Pitman, Paris (1994) 13-51.

[7] D. Braess and W. Dahmen, Stability estimates of the mortar finite element method for 3-dimensional problems. East-West J. Numer. Math. 6 (1998) 249-264.

[8] D. Braess, W. Dahmen and C. Wieners, A multigrid algorithm for the mortar finite element method. SIAM J. Numer. Anal. 37 (1999) 48-69.

[9] S.C. Brenner and L.R. Scott, The Mathematical Theory of Finite Element Methods. Springer-Verlag, New York (1994).

[10] F. Brezzi and M. Fortin, Mixed and hybrid finite element methods. Springer-Verlag, New York (1991).

[11] F. Brezzi and D. Marini, Error estimates for the three-field formulation with bubble stabilization. Math. Comp 70 (2001) 911-934.

[12] F. Brezzi, L. Franca, D. Marini and A. Russo, Stabilization techniques for domain decomposition methods with non-matching grids, in Proc. of the 9th International Conference on Domain Decomposition, P. Bjørstad, M. Espedal and D. Keyes Eds., Domain Decomposition Press, Bergen (1998) 1-11.

[13] A. Buffa, Error estimate for a stabilised domain decomposition method with nonmatching grids. Numer. Math. 90 (2002) 617-640.

[14] J. Gopalakrishnan, On the mortar finite element method. Ph.D. thesis, Texas A\&M University (1999).

[15] C. Kim, R.D. Lazarov, J.E. Pasciak and P.S. Vassilevski, Multiplier spaces for the mortar finite element method in three dimensions. SIAM J. Numer. Anal. 39 (2000) 519-538.

[16] B.P. Lamichhane and B.I. Wohlmuth, Higher order dual Lagrange multiplier spaces for mortar finite element discretizations. CALCOLO 39 (2002) 219-237.

[17] P. Seshaiyer and M. Suri, Uniform hp convergence results for the mortar finite element method. Math. of Comput. 69 (2000) $521-546$.

[18] R. Stevenson, Locally supported, piecewise polynomial biorthogonal wavelets on non-uniform meshes. Constr. Approx. 19 (2003) 477-508.

[19] C. Wieners and B.I. Wohlmuth, The coupling of mixed and conforming finite element discretizations, in Proc. of the 10th International Conference on Domain Decomposition, J. Mandel, C. Farhat and X. Cai Eds., AMS, Contemp. Math. (1998) $546-553$.

[20] C. Wieners and B.I. Wohlmuth, Duality estimates and multigrid analysis for saddle point problems arising from mortar discretizations. SISC 24 (2003) 2163-2184.

[21] B.I. Wohlmuth, Discretization Methods and Iterative Solvers Based on Domain Decomposition. Lect. Notes Comput. Sci. 17, Springer, Heidelberg (2001)

[22] B.I. Wohlmuth and R.H. Krause, Multigrid methods based on the unconstrained product space arising from mortar finite element discretizations. SIAM J. Numer. Anal. 39 (2001) 192-213. 